\section{Monosaccharides: A ToF-SIMS reference spectra database. I. Negative polarity}

\author{
Laetitia Bernard, Rowena Crockett, and Maciej Kawecki ${ }^{\text {a) }}$ \\ Laboratory of Nanoscale Materials Science, Empa, CH-8600 Dübendorf, Switzerland
}

(Received 20 August 2019; accepted 30 October 2019; published 3 December 2019)

\begin{abstract}
The number of time-of-flight secondary ion mass spectrometry studies on biological tissues and cells has significantly increased since the development of primary ion sources that allow not only elemental but also molecular analysis. Substantial fragmentation during ionic bombardment results in a large number of peaks, rendering data analysis complex. Complete and trustable sets of reference spectra for the main biological building blocks, i.e., amino acids, monosaccharides, fatty acids, and nucleotides, are required. This work aims to provide an accurate and extensive library of reference spectra for monosaccharides, measured with the $\mathrm{Bi}_{3}{ }^{+}$primary ion. Here (Paper I), the negative polarity spectra and lists of associated characteristic fragments are presented. Published by the AVS. https://doi.org/10.1116/1.5125102
\end{abstract}

Keywords: ToF-SIMS, carbohydrate, sugar, monosaccharide, mass spectrometry, fragmentation
Accession \#s: 01573, 01574,

01575, 01576, 01577, 01578,

01579, 01580, 01581, 01582,

$01583,01584,01585,01586$,

$01587,01588,01589,01590$

01591

Technique: SIMS

Host Material: Silicon (100) wafer

Instrument: IONTOF TOF-SIMS.5

Major Species in Spectra: $\mathrm{C}, \mathrm{H}, \mathrm{O}$, (N)

Minor Species in Spectra: $\mathrm{Cl}$

Published Spectra: 19

Spectra in Electronic Record: 19

Published Figures: 20

Spectral Category: Reference

\section{INTRODUCTION}

Monosaccharides are the building blocks of structural polymers of both plant [e.g., cellulose, pectins, and hemicelluloses (Refs. 1 and 2)] and animal origin [e.g., chitin (Refs. 3 and 4)] and are essential components of glycoproteins (Refs. 5-7). Monosaccharides, their derivatives, and polymers are also indispensable in cellular respiration (Ref. 8) and for energy storage and transport in biological organisms (Refs. 9 and 10). Further, specific monosaccharides and monosaccharide derivatives act as secondary messengers [e.g., inositol 1,4,5-triphosphate in $\mathrm{Ca}^{2+}$ release (Ref. 11)], as essential reagents in detoxification [e.g., glucuronic acid in phase II liver metabolism (Ref. 12)], and as markers mediating cell-cell interaction (Ref. 13).

A ToF-SIMS study of seven selected hexoses was presented by Berman et al. based on the analysis of positive secondary ions produced by the bombardment with $\mathrm{Au}+$ primary ions (Ref. 14). They could demonstrate the fragmentation pathway of glucose via the loss of successive water molecules. Interestingly, they also found that different isomers undergo different fragmentation patterns, which shows that ToF-SIMS is a powerful tool to obtain an accurate differentiation of monosaccharides. The work presented here provides a library of ToF-SIMS reference spectra for the following monosaccharides: allose, arabinose, fructose, fucose, galactose, 2-deoxygalactose, galacturonic acid, glucose, glucuronic acid, mannose, myoinositol, rhamnose, ribose, 2-deoxyribose, xylose, $\quad N$-acetylgalactosamine, $\quad N$-acetylglucosamine, $N$-acetylmuramic acid, and $N$-acetylneuraminic acid. Characteristic fragments are listed beside the spectra for each monosaccharide. This part (Paper I) of the monosaccharide database contains the negative polarity spectra. The positive spectra are published in Paper II (Ref. 15). Analog databases for the full set of proteinogenic amino acids + glycine (Refs. 16 and 17) are available. Peak lists (Ref. 18) and reference spectra (Ref. 19) for selected lipids can be found in the literature.

The monosaccharides were purchased in the form of pure powders. D-Galacturonic acid and D-glucuronic acid were from

a)Electronic mail: maciej.kawecki@empa.ch
Fluka and all others from Sigma Aldrich. Each powder was dissolved in pure $\mathrm{H}_{2} \mathrm{O}$ (Sigma Aldrich, 270733) at a concentration of $0.1 \mathrm{M}$. Host silicon wafers of $1 \times 1 \mathrm{~cm}^{2}$ size were first washed by submersion in subsequent ultrasound baths of $2 \times 15 \mathrm{~min}$ in acetone and $1 \times 15 \mathrm{~min}$ in ethanol and then plasma treated for a duration of $10 \mathrm{~min}$ to render the surface hydrophilic. $50 \mu \mathrm{l}$ of sample solution was then deposited on the pretreated silicon wafer substrates, and the water was allowed to evaporate in a fume hood at room temperature.

The spectra were acquired on a ToF-SIMS 5 instrument (IONTOF GmbH) with a $25 \mathrm{keV} \mathrm{Bi}_{3}{ }^{+}$primary ion beam operated in high mass resolution spectral mode. The primary ion dose density was kept below the static limit $\left(\leq 10^{12}\right.$ ions $\left./ \mathrm{cm}^{2}\right)$. Four to ten randomly selected areas of $200 \times 200 \mu \mathrm{m}^{2}$ $\left(128 \times 128\right.$ pxls $^{2}, 50$ scans $)$ were analyzed for each monosaccharide sample to insure good reproducibility. No electron flood gun was used. All spectra were normalized to their respective total ions count. The most relevant characteristic fragments are displayed directly on the spectra and reported in the tables. Intact monosaccharide molecules are referred to as " $M$," and contaminants as "c." Major peaks, where a unique attribution was not possible, e.g., due to overlap beyond the mass resolution of two potential fragmentation products, are marked with "n.a." standing for not (uniquely) attributed. The deviation values for all assigned peaks are below $150 \mathrm{ppm}$. The peak assignments to the fragmentation species given in the tables are tentative, and the smaller fragments are nonspecific.

Raw data (ASCII) are included in the supplementary material (Ref. 20).

\section{SPECIMEN DESCRIPTION (ACCESSION \#: 01573, 01574, 01575, 01576, 01577, 01578, 01579, 01580, 01581, 01582, 01583, 01584, 01585, 01586, 01587, 01588, 01589, 01590, 01591)}

Host Material: Silicon (100) wafer

CAS Registry \#: D-Allose: 2595-97-3, L-arabinose: 87-72-9, D-fructose: 57-48-7, D-fucose: 3615-37-0, D-galactose: 59-23-4, 2-deoxy-D-galactose: 1949-89-9, D-galacturonic acid: 91510-52-2, D-glucose: 50-99-7, D-glucoronic acid: 6556-12-3, D-mannose: 3458-28-4, myoinositol: 87-89-8, L-rhamnose 
monohydrate: 10030-85-0, D-ribose: 50-69-1, 2-deoxy-D-ribose: 533-67-5, D-xylose: 58-86-6, $N$-acetyl-D-galactosamine: 1811-31-0, $N$-acetyl-D-glucosamine: 7512-17-6, $N$-acetylmuramic acid: 10597-89-4, $N$-acetylneuraminic acid: $131-48-6$

Host Material Characteristics: Homogeneous; solid; single crystal; semiconductor

Chemical Name: D-Allose (01573), L-arabinose (01574), D-fructose (01575), D-fucose (01576), D-galactose (01577), 2-deoxy-D-galactose (01578), D-galacturonic acid (01579), D-glucose (01580), D-glucuronic acid (01581), D-mannose (01582), myoinositol (01583), L-rhamnose (01584), D-ribose (01585), 2-deoxy-D-ribose (01586), D-xylose (01587), $\mathrm{N}$-acetyl-D-galactosamine (01588), $\quad \mathrm{N}$-acetyl-D-glucosamine (01589), $N$-acetylmuramic acid (01590), $N$-acetylneuraminic acid (015891)

Source: Sigma Aldrich and Fluka

Host Composition: ${ }_{n o x} \mathrm{SiO}_{2} / \mathrm{SiO}_{2}$

Form: Pure powder dissolved in pure $\mathrm{H}_{2} \mathrm{O}$ (Sigma Aldrich, 270733), sonicated and subsequently drop-deposited on the host wafer

Lot No.: Not specified

Structure: Allose: $\mathrm{C}_{6} \mathrm{H}_{12} \mathrm{O}_{6}$, arabinose: $\mathrm{C}_{5} \mathrm{H}_{10} \mathrm{O}_{5}$, fructose: $\mathrm{C}_{6} \mathrm{H}_{12} \mathrm{O}_{6}$, fucose: $\mathrm{C}_{6} \mathrm{H}_{12} \mathrm{O}_{5}$, galactose: $\mathrm{C}_{6} \mathrm{H}_{12} \mathrm{O}_{6}$, 2-deoxygalactose: $\mathrm{C}_{6} \mathrm{H}_{12} \mathrm{O}_{5}$, galacturonic acid: $\mathrm{C}_{6} \mathrm{H}_{10} \mathrm{O}_{7}$, glucose: $\mathrm{C}_{6} \mathrm{H}_{12} \mathrm{O}_{6}$, glucoronic acid: $\mathrm{C}_{6} \mathrm{H}_{10} \mathrm{O}_{7}$, mannose: $\mathrm{C}_{6} \mathrm{H}_{12} \mathrm{O}_{6}$, myoinositol: $\mathrm{C}_{6} \mathrm{H}_{12} \mathrm{O}_{6}$, rhamnose: $\mathrm{C}_{6} \mathrm{H}_{12} \mathrm{O}_{5}$, ribose: $\mathrm{C}_{5} \mathrm{H}_{10} \mathrm{O}_{5}, 2-$ deoxyribose: $\mathrm{C}_{5} \mathrm{H}_{10} \mathrm{O}_{4}$, xylose: $\mathrm{C}_{5} \mathrm{H}_{10} \mathrm{O}_{5}, N$-acetylgalactosamine: $\mathrm{C}_{8} \mathrm{H}_{15} \mathrm{NO}_{6}, N$-acetylglucosamine: $\mathrm{C}_{8} \mathrm{H}_{15} \mathrm{NO}_{6}, N$-acetylmuramic acid: $\mathrm{C}_{11} \mathrm{H}_{19} \mathrm{NO}_{8}, N$-acetylneuraminic acid: $\mathrm{C}_{11} \mathrm{H}_{19} \mathrm{NO}_{9}$

History and Significance: As such and/or combined to other small molecules, monosaccharides play various essential roles in the structure and metabolic functions of living organisms.

As Received Condition: Pure powders in glass vials

Analyzed Region: 4-10 randomly selected regions of $200 \times 200 \mu \mathrm{m}^{2}$

Ex Situ Preparation/Mounting: $50 \mu \mathrm{l}$ of $0.1 \mathrm{M}$ monosaccharide solutions, prepared in pure $\mathrm{H}_{2} \mathrm{O}$ (Sigma Aldrich, 270733), were drop-deposited on $1 \times 1 \mathrm{~cm}^{2}$ plasma-treated $\mathrm{Si}$ (100) substrates, in a fume hood. Full coverage of the solutions on each wafer surface was assured. The samples were left in the fume hood for drying and then directly inserted in the UHV chamber and analyzed.

In Situ Preparation: None

Charge Control: None

Temp. During Analysis: $300 \mathrm{~K}$

Pressure During Analysis: $5.6 \times 10^{-8} \mathrm{~Pa}$

Preanalysis Beam Exposure: None

\section{INSTRUMENT CONFIGURATION}

Manufacturer and Model: IONTOF TOF-SIMS.5

Analyzer Type: Time-of-flight

Detector Type: Microchannel plate

Experiment Type: Mass spectra

Sample Rotation: None
Oxygen Flood Source: None

Other Flood Source: None

Unique Instrument Features Used: None

Energy Acceptance Window: $20 \mathrm{eV}$

Postacceleration Voltage: $10000 \mathrm{eV}$

Sample Bias: $0 \mathrm{eV}$

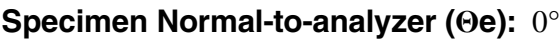

\section{Ion Sources}

Ion Source \# 1 of 1

Purpose of this Ion Source: Analysis beam

Ion Source Manufacturer: IONTOF (Münster, Germany)

Ion Source Model: $25 \mathrm{keV} \mathrm{Bi/Mn} \mathrm{emitter}$

Beam Mass Filter: Electrodynamic mass filter

Beam Species and Charge State: $\mathrm{Bi}_{3}{ }^{+}$

Beam Gating Used: None

Additional Beam Comments: None

Beam Voltage: $25000 \mathrm{eV}$

Net Beam Voltage (impact voltage): $25000 \mathrm{eV}$

Ion Pulse Width: $17.2 \mathrm{~ns}$

Ion Pulse Rate: $10 \mathrm{kHz}$

Pulsed Beam Current: 0.000 74-0.000 79 nA

Current Measurement Method: Faraday cup

Beam Diameter: N/A $(\mu \mathrm{m})$

Beam Raster Size: $200 \times 200 \mu \mathrm{m}^{2}$

Beam Incident Angle: $45^{\circ}$

Source-to-Analyzer Angle: $45^{\circ}$

\section{ACKNOWLEDGMENT}

The authors are thankful for the funding received from the Swiss National Science Foundation through Grant No. CR23I2-162828.

\section{REFERENCES}

1. H. Höfte and A. Voxeur, Curr. Biol. 27, 853 (2017).

2. H. Zhu, W. Luo, P. N. Ciesielski, Z. Fang, J. Y. Zhu, G. Henriksson, M. E. Himmel, and L. Hu, Chem. Rev. 116, 9305 (2016).

3. H.-O. Fabritius, C. Sachs, P. R. Triguero, and D. Raabe, Adv. Mater. 21, 391 (2009).

4. N. V. Majeti and R. Kumar, React. Funct. Polym. 46, 1 (2000).

5. C. G. Gahmberg and M. Tolvanen, Trends Bichem. Sci. 21, 308 (1996).

6. G. J. Strous and J. Dekker, Crit. Rev. Biochem. Mol. Biol. 27, 57 (1992).

7. E. Maverakis, K. Kim, M. Shimoda, M. E. Gershwin, F. Patel, R. Wilken, S. Raychaudhuri, L. R. Ruhaak, and C. B. Lebrilla, J. Autoimmun. 57, 1 (2015).

8. B. Alberts, A. Johnson, J. Lewis, D. Morgan, M. Raff, K. Roberts, and P. Walter, Molecular Biology of the Cell, 6th ed. (Garland Science, New York, 2008), Chap. 2. 
9. S. C. Zeeman, J. Kossmann, and A. M. Smith, Annu. Rev. Plant. Biol. 61, 209 (2010).

10. D. H. Wasserman, Am. J. Physiol. Endocrinol. Metab. 296, E11 (2009).

11. H. Streb, R. F. Irvine, M. J. Berridge, and I. Schulz, Nature 306, 67 (1983).

12. G. J. F. Dutton, Glucuronidation of Drugs and Other Compounds (CRC, Boca Raton, FL, 1980).

13. R. L. Schnaar, Arch. Biochem. Biophys. 426, 163 (2004).

14. E. S. F. Berman, K. S. Kulp, M. G. Knize, L. Wu, E. J. Nelson, D. O. Nelson, and K. J. Wu, Anal. Chem. 78, 6497 (2006).
15. L. Bernard, R. Crockett, and M. Kawecki, Surf. Sci. Spectra 26, 025002 (2019).

16. M. Kawecki and L. Bernard, Surf. Sci. Spectra 25, 015001 (2018).

17. M. Kawecki and L. Bernard, Surf. Sci. Spectra 25, 015002 (2018).

18. M. K. Passarelli and N. Winograd, Biochim. Biophys. Acta 1811, 976 (2011).

19. M. J. Taylor, K. Y. Zhang, D. J. Graham, and L. J. Gamble, Surf. Sci. Spectra 25, 025001 (2019).

20. See supplementary material at https://doi.org/10.1116/ 1.5125102 for the raw data exported in ASCII. 


\begin{tabular}{lccc}
\hline & & D-ALLOSE & \\
\hline Spectrum ID \# & Mass (Da) & Species & Peak Assignment \\
\hline 01573 & 71.014 & $\mathrm{C}_{3} \mathrm{H}_{3} \mathrm{O}_{2}^{-}$ & $\mathrm{C} 1$ to $\mathrm{C}$ fragment of $\mathrm{M}-\mathrm{H}_{2} \mathrm{O}$ \\
$\ldots$ & 87.009 & $\mathrm{C}_{3} \mathrm{H}_{3} \mathrm{O}_{3}^{-}$ & $\mathrm{C} 1$ to $\mathrm{C} 3$ fragment of $\mathrm{M}-2 \mathrm{H}$ \\
$\ldots$ & 99.009 & $\mathrm{C}_{4} \mathrm{H}_{3} \mathrm{O}_{3}^{-}$ & $\mathrm{C} 1$ to $\mathrm{C} 4$ fragment of $\mathrm{M}-\mathrm{H}_{2} \mathrm{O}-2 \mathrm{H}$ \\
$\ldots$ & 119.035 & $\mathrm{C}_{4} \mathrm{H}_{7} \mathrm{O}_{4}^{-}$ & $\mathrm{C}$ to $\mathrm{C} 6$ fragment of $\mathrm{M}-2 \mathrm{H}$ \\
$\ldots$ & 141.019 & $\mathrm{C}_{6} \mathrm{H}_{5} \mathrm{O}_{4}^{-}$ & $\mathrm{M}-2\left(\mathrm{H}_{2} \mathrm{O}\right)-3 \mathrm{H}$ \\
$\ldots$ & 159.030 & $\mathrm{C}_{6} \mathrm{H}_{7} \mathrm{O}_{5}^{-}$ & $\mathrm{M}-\left(\mathrm{H}_{2} \mathrm{O}\right)-3 \mathrm{H}$ \\
$\ldots$ & 177.040 & $\mathrm{C}_{6} \mathrm{H}_{9} \mathrm{O}_{6}^{-}$ & $\mathrm{M}-3 \mathrm{H}$ \\
$\ldots$ & 179.056 & $\mathrm{C}_{6} \mathrm{H}_{11} \mathrm{O}^{-}$ & $\mathrm{M}-\mathrm{H}$ \\
\hline
\end{tabular}

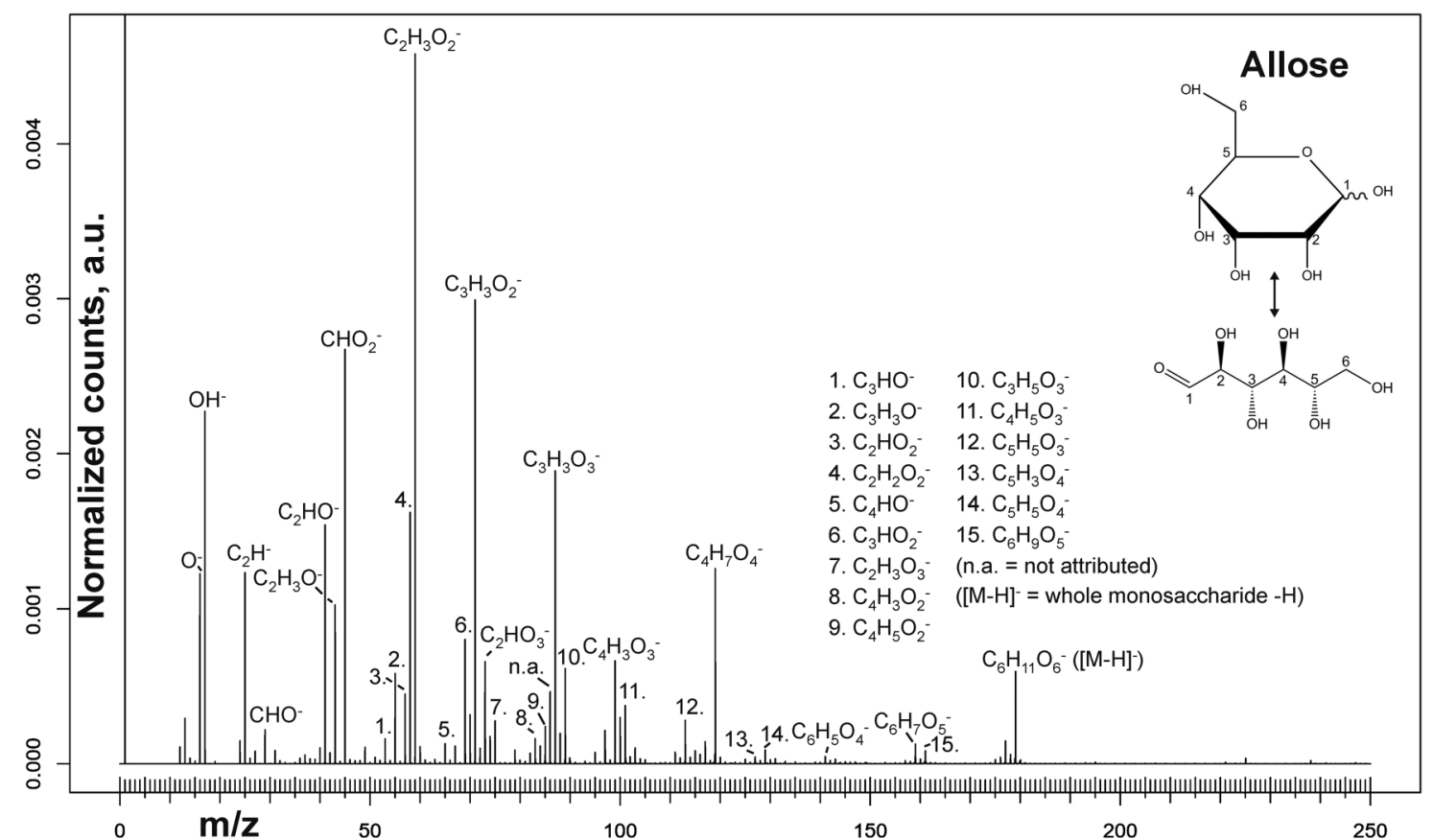

Accession \#

Host Material:

Technique:

Secondary Source Polarity:

Mass Range:

Species Used for Mass

Calibration:

Primary lon Dose:

Primary Ion Pulse Width:

Pulsed Beam Current:

Biological significance:

\section{3-01}

Silicon wafer

SIMS

Negative

0-250 Da

$\mathrm{C}^{-}, \mathrm{CH}^{-}, \mathrm{CH}_{2}^{-}, \mathrm{CH}_{3} \mathrm{O}^{-}, \mathrm{C}_{4} \mathrm{H}_{3}^{-}, \mathrm{C}_{3} \mathrm{HO}^{-}, \mathrm{C}_{2} \mathrm{HO}_{2}^{-}$

$1.01 \times 10^{12} \mathrm{~cm}^{-2}$

$17.2 \mathrm{~ns}$

$0.00079 \mathrm{nA}$

D-Allose exists only in small amounts in nature 


\begin{tabular}{lccc}
\hline & & L-ARABINOSE & \\
\hline Spectrum ID \# & Mass (Da) & Species & Peak Assignment \\
\hline 01574 & 71.014 & $\mathrm{C}_{3} \mathrm{H}_{3} \mathrm{O}_{2}^{-}$ & $\mathrm{C} 1$ to $\mathrm{C} 3$ fragment of $\mathrm{M}-\mathrm{H}_{2} \mathrm{O}$ \\
$\ldots$ & 87.009 & $\mathrm{C}_{3} \mathrm{H}_{3} \mathrm{O}_{3}^{-}$ & $\mathrm{C} 1$ to $\mathrm{C}$ fragment of $\mathrm{M}-2 \mathrm{H}$ \\
$\ldots$ & 89.024 & $\mathrm{C}_{3} \mathrm{H}_{5} \mathrm{O}_{3}^{-}$ & $\mathrm{C} 1$ to $\mathrm{C}$ fragment of $\mathrm{M}$ \\
$\ldots$ & 99.009 & $\mathrm{C}_{4} \mathrm{H}_{3} \mathrm{O}_{3}^{-}$ & $\mathrm{C} 1$ to $\mathrm{C} 4$ fragment of $\mathrm{M}-\mathrm{H}_{2} \mathrm{O}-2 \mathrm{H}$ \\
$\ldots$ & 129.019 & $\mathrm{C}_{5} \mathrm{H}_{5} \mathrm{O}_{4}^{-}$ & $\mathrm{M}-\left(\mathrm{H}_{2} \mathrm{O}\right)-3 \mathrm{H}$ \\
$\ldots$ & 149.046 & $\mathrm{C}_{5} \mathrm{H}_{9} \mathrm{O}_{5}^{-}$ & $\mathrm{M}-\mathrm{H}$ \\
$\ldots$ & 299.098 & $\mathrm{C}_{10} \mathrm{H}_{19} \mathrm{O}_{10}^{-}$ & $2 \mathrm{M}-\mathrm{H}$ \\
\hline
\end{tabular}

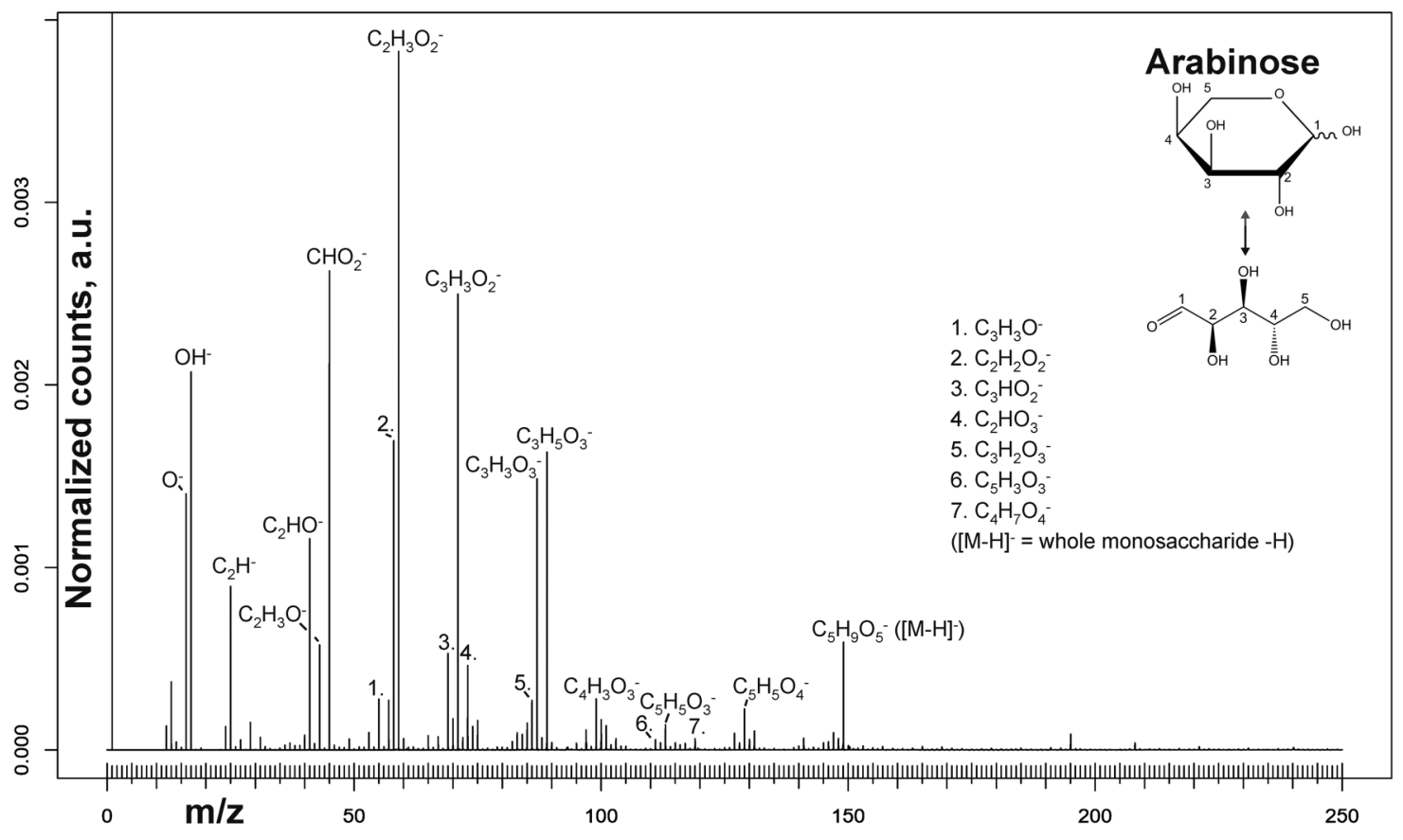

\begin{tabular}{|c|c|}
\hline Accession \# & 01574-01 \\
\hline Host Material: & Silicon wafer \\
\hline$\square$ Technique: & SIMS \\
\hline $\begin{array}{r}\text { Secondary Source } \\
\text { Polarity: }\end{array}$ & Negative \\
\hline Mass Range: & $0-250 \mathrm{Da}$ \\
\hline $\begin{array}{r}\text { Species Used for Mass } \\
\text { Calibration: }\end{array}$ & $\mathrm{C}^{-}, \mathrm{CH}^{-}, \mathrm{CH}_{2}^{-}, \mathrm{CH}_{3} \mathrm{O}^{-}, \mathrm{C}_{4} \mathrm{H}_{3}^{-}, \mathrm{C}_{3} \mathrm{HO}^{-}, \mathrm{C}_{2} \mathrm{HO}_{2}^{-}$ \\
\hline Primary lon Dose: & $9.46 \times 10^{11} \mathrm{~cm}^{-2}$ \\
\hline $\begin{array}{r}\text { Primary Ion Pulse } \\
\text { Width: }\end{array}$ & $17.2 \mathrm{~ns}$ \\
\hline Pulsed Beam Current: & $0.00074 \mathrm{nA}$ \\
\hline Biological significance: & $\begin{array}{l}\text { L-Arabinose is found in a range of plant polysaccharides, e.g., the } \\
\text { plant cell wall heteropolysaccharides pectin and hemicellulose }\end{array}$ \\
\hline
\end{tabular}




\begin{tabular}{|c|c|c|c|}
\hline \multicolumn{4}{|c|}{ D-FRUCTOSE } \\
\hline Spectrum ID \# & Mass (Da) & Species & Peak Assignment \\
\hline 01575 & 71.014 & $\mathrm{C}_{3} \mathrm{H}_{3} \mathrm{O}_{2}^{-}$ & $\mathrm{C} 1$ to $\mathrm{C} 3$ fragment of $\mathrm{M}-\mathrm{H}_{2} \mathrm{O}$ \\
\hline$\ldots$ & 75.009 & $\mathrm{C}_{2} \mathrm{H}_{3} \mathrm{O}_{3}^{-}$ & $\ldots$ \\
\hline$\ldots$ & 87.009 & $\mathrm{C}_{3} \mathrm{H}_{3} \mathrm{O}_{3}^{-}$ & $\mathrm{C} 1$ to $\mathrm{C} 3$ fragment of $\mathrm{M}-2 \mathrm{H}$ \\
\hline$\ldots$ & 89.024 & $\mathrm{C}_{3} \mathrm{H}_{5} \mathrm{O}_{3}^{-}$ & C1 to C3 fragment of M \\
\hline$\ldots$ & 99.009 & $\mathrm{C}_{4} \mathrm{H}_{3} \mathrm{O}_{3}^{-}$ & $\mathrm{C} 1$ to $\mathrm{C} 4$ fragment of $\mathrm{M}-\mathrm{H}_{2} \mathrm{O}-2 \mathrm{H}$ \\
\hline$\ldots$ & 113.024 & $\mathrm{C}_{5} \mathrm{H}_{5} \mathrm{O}_{3}^{-}$ & $\ldots$ \\
\hline$\ldots$ & 117.019 & $\mathrm{C}_{4} \mathrm{H}_{5} \mathrm{O}_{4}^{-}$ & $\mathrm{C} 1$ to $\mathrm{C} 4$ fragment of $\mathrm{M}-2 \mathrm{H}$ \\
\hline$\ldots$ & 129.019 & $\mathrm{C}_{5} \mathrm{H}_{5} \mathrm{O}_{4}^{-}$ & $\mathrm{M}-\left(\mathrm{CH}_{3} \mathrm{O}\right)-\left(\mathrm{H}_{2} \mathrm{O}\right)-2 \mathrm{H}$ \\
\hline$\ldots$ & 159.030 & $\mathrm{C}_{6} \mathrm{H}_{7} \mathrm{O}_{5}^{-}$ & $\mathrm{M}-\left(\mathrm{H}_{2} \mathrm{O}\right)-3 \mathrm{H}$ \\
\hline$\ldots$ & 178.048 & $\mathrm{C}_{6} \mathrm{H}_{10} \mathrm{O}_{6}^{-}$ & $\mathrm{M}-2 \mathrm{H}$ \\
\hline$\ldots$ & 179.056 & $\mathrm{C}_{6} \mathrm{H}_{11} \mathrm{O}_{6}^{-}$ & $\mathrm{M}-\mathrm{H}$ \\
\hline$\ldots$ & 359.120 & $\mathrm{C}_{12} \mathrm{H}_{23} \mathrm{O}_{12}^{-}$ & $2 \mathrm{M}-\mathrm{H}$ \\
\hline
\end{tabular}

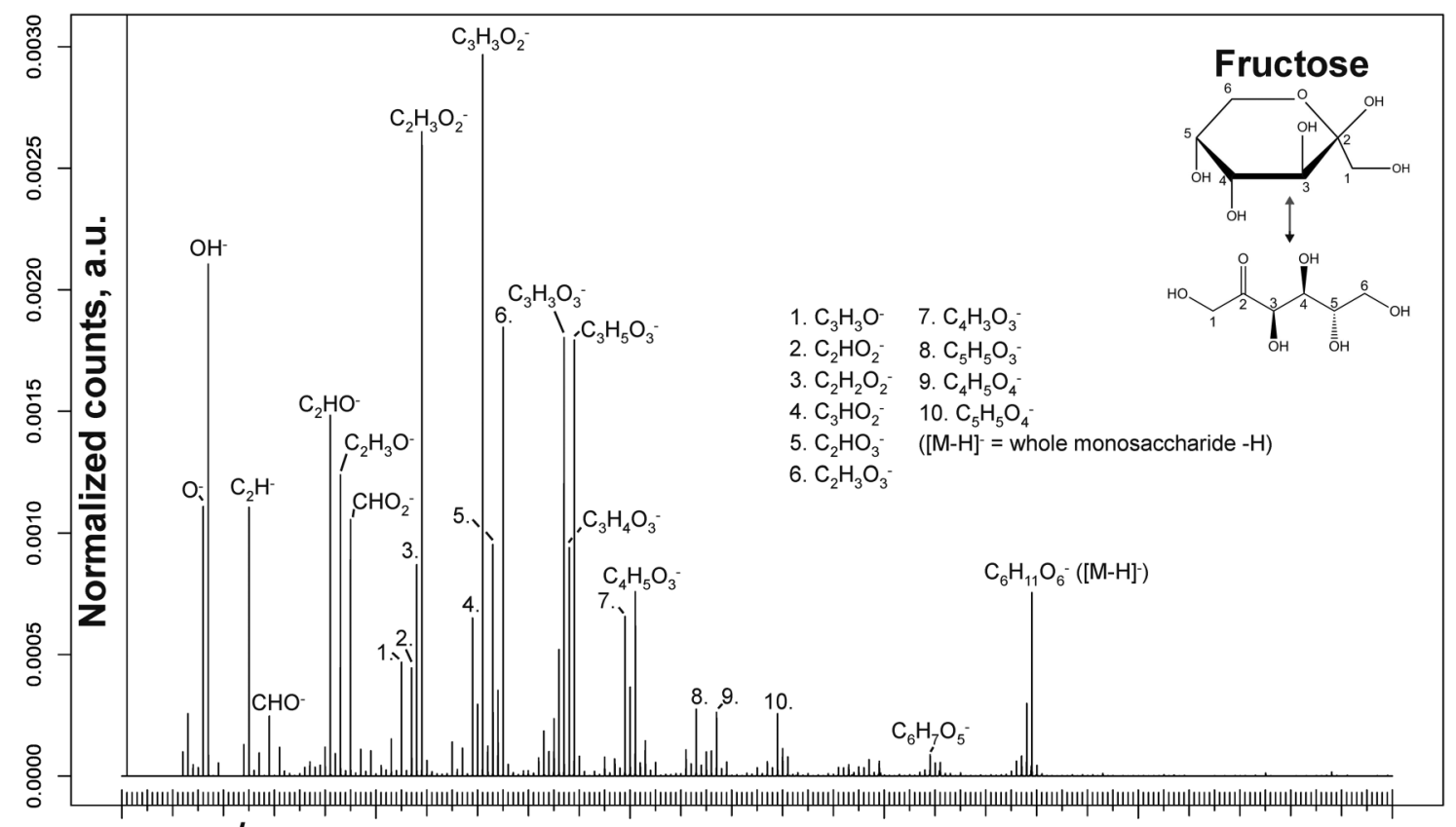

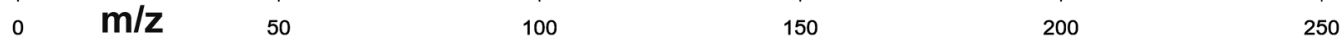

\begin{tabular}{rc}
\hline Accession \# & $01575-01$ \\
\hline Host Material: & Silicon wafer \\
Technique: & SIMS \\
Negative \\
Mass Range: & $0-250 \mathrm{Da}$ \\
Secondary Source Polarity: & $\mathrm{C}^{-}, \mathrm{CH}^{-}, \mathrm{CH}_{2}^{-}, \mathrm{CH}_{3} \mathrm{O}^{-}, \mathrm{C}_{4} \mathrm{H}_{3}^{-}, \mathrm{C}_{3} \mathrm{HO}^{-}, \mathrm{C}_{2} \mathrm{HO}_{2}{ }^{-}$ \\
Calibration: & $1.01 \times 10^{12} \mathrm{~cm}^{-2}$ \\
Species Used for Mass & $17.2 \mathrm{~ns}^{-}$ \\
Primary lon Dose: & $0.00079 \mathrm{nA}$ \\
Primary lon Pulse Width: & D-Fructose is important for plant metabolism and energy storage, where, e.g., \\
Pulsed Beam Current: & sucrose (a.k.a. table sugar) is a disaccharide composed of one fructose and one \\
Biological significance: & glucose unit. D-Fructose-based polysaccharides, fructans, are important storage \\
& macromolecules but also function as cryoprotectants in plants regularly exposed \\
& to low temperatures and reduce membrane leakage in periods drought \\
\hline
\end{tabular}




\section{D-FUCOSE}

\begin{tabular}{lrcc}
\hline Spectrum ID \# & Mass (Da) & Species & Peak Assignment \\
\hline 01576 & 71.014 & $\mathrm{C}_{3} \mathrm{H}_{3} \mathrm{O}_{2}^{-}$ & $\mathrm{C} 1$ to $\mathrm{C} 3$ fragment of $\mathrm{M}-2 \mathrm{H}$ \\
$\ldots$ & 85.030 & $\mathrm{C}_{4} \mathrm{H}_{5} \mathrm{O}_{2}^{-}$ & $\mathrm{C} 1$ to $\mathrm{C} 4$ fragment of $\mathrm{M}-\left(\mathrm{H}_{2} \mathrm{O}\right)$ \\
$\ldots$ & 87.009 & $\mathrm{C}_{3} \mathrm{H}_{3} \mathrm{O}_{3}^{-}$ & pyruvate \\
$\ldots$ & 99.009 & $\mathrm{C}_{4} \mathrm{H}_{3} \mathrm{O}_{3}^{-}$ & $\mathrm{C} 1$ to $\mathrm{C} 4$ fragment of $\mathrm{M}-4 \mathrm{H}$ \\
$\ldots$ & 127.004 & $\mathrm{C}_{5} \mathrm{H}_{3} \mathrm{O}_{4}^{-}$ & $\ldots$ \\
$\ldots$ & 141.019 & $\mathrm{C}_{6} \mathrm{H}_{5} \mathrm{O}_{4}^{-}$ & $\ldots$ \\
$\ldots$ & 163.061 & $\mathrm{C}_{6} \mathrm{H}_{11} \mathrm{O}_{5}^{-}$ & $\mathrm{M}-\mathrm{H}$ \\
\hline
\end{tabular}

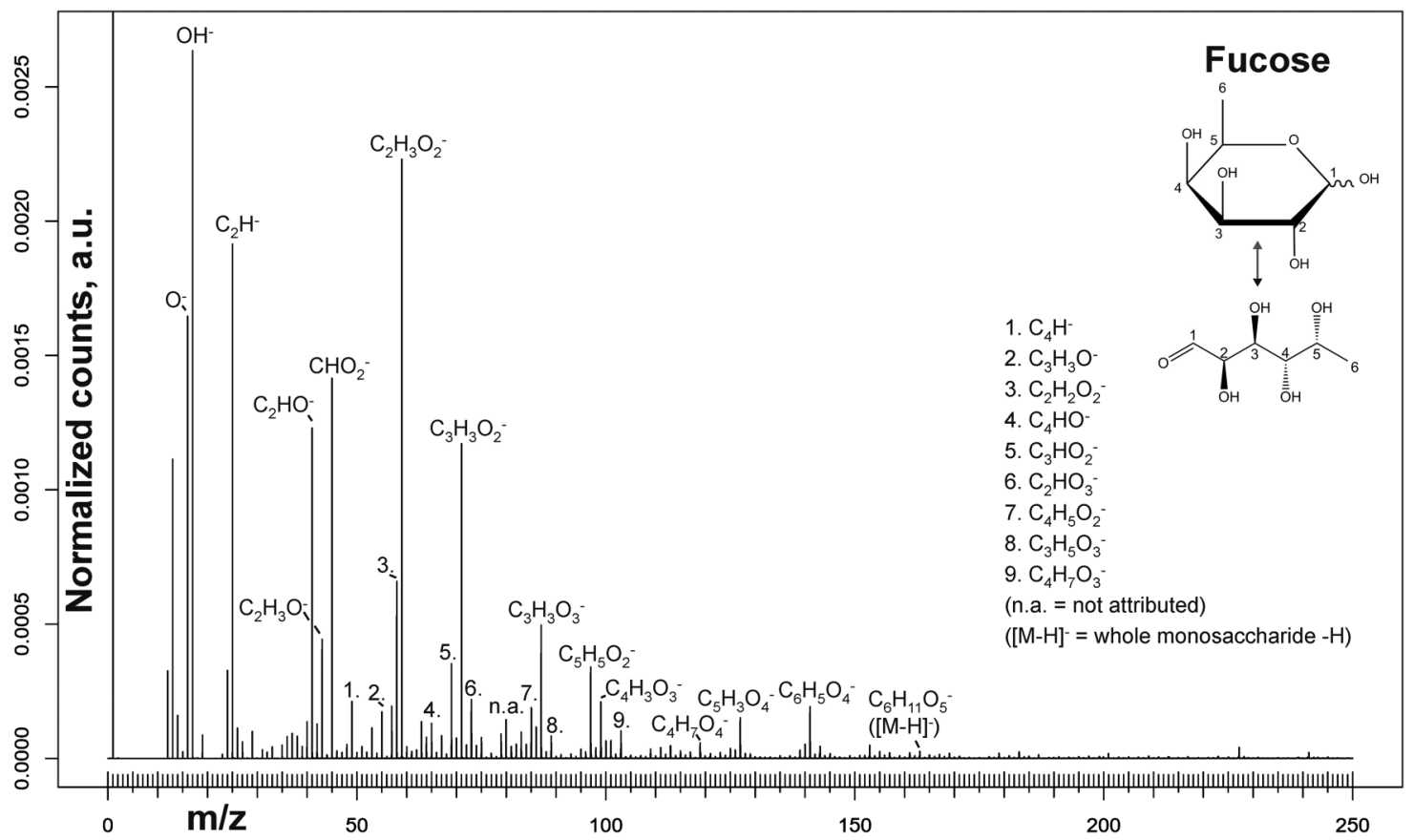

\begin{tabular}{rc}
\hline Accession \# & $01576-01$ \\
\hline Host Material: \\
Technique: & Silicon wafer \\
Mass Range: & SIMS \\
Segative \\
Secondary Source Polarity: & $0-250 \mathrm{Da}$ \\
Species Used for Mass Calibration: & $\mathrm{C}^{-}, \mathrm{CH}^{-}, \mathrm{CH}_{2}^{-}, \mathrm{CH}_{3} \mathrm{O}^{-}, \mathrm{C}_{4} \mathrm{H}_{3}^{-}, \mathrm{C}_{3} \mathrm{HO}^{-}, \mathrm{C}_{2} \mathrm{HO}_{2}{ }^{-}$ \\
Primary lon Dose: & $9.46 \times 10^{11} \mathrm{~cm}^{-2}$ \\
Primary lon Pulse Width: & $17.2 \mathrm{~ns}$ \\
Pulsed Beam Current: & $0.00074 \mathrm{nA}$ \\
Biological significance: & L-Fucose is found in a wide range of mucoproteins and many \\
& membrane glycoproteins and glycolipids (it is, e.g., part of the \\
& H-antigen, the base unit of the membrane structure that defines the \\
& human blood type) \\
\hline
\end{tabular}




\begin{tabular}{lccc}
\hline & & D-GALACTOSE & \\
\hline Spectrum ID \# & Mass (Da) & Species & Peak Assignment \\
\hline 01577 & 71.014 & $\mathrm{C}_{3} \mathrm{H}_{3} \mathrm{O}_{2}^{-}$ & $\mathrm{C} 1$ to $\mathrm{C}$ fragment of $\mathrm{M}-\mathrm{H}_{2} \mathrm{O}$ \\
$\ldots$ & 87.009 & $\mathrm{C}_{3} \mathrm{H}_{3} \mathrm{O}_{3}^{-}$ & $\mathrm{C} 1$ to $\mathrm{C}$ fragment of $\mathrm{M}-2 \mathrm{H}$ \\
$\ldots$ & 99.009 & $\mathrm{C}_{4} \mathrm{H}_{3} \mathrm{O}_{3}^{-}$ & $\mathrm{C} 1$ to $\mathrm{C}$ fragment of $\mathrm{M}-\mathrm{H}_{2} \mathrm{O}-2 \mathrm{H}$ \\
$\ldots$ & 119.035 & $\mathrm{C}_{4} \mathrm{H}_{7} \mathrm{O}_{4}^{-}$ & $\mathrm{C}$ to $\mathrm{C}$ fragment of $\mathrm{M}-2 \mathrm{H}$ \\
$\ldots$ & 141.019 & $\mathrm{C}_{6} \mathrm{H}_{5} \mathrm{O}_{4}^{-}$ & $\mathrm{M}-2\left(\mathrm{H}_{2} \mathrm{O}\right)-3 \mathrm{H}$ \\
$\ldots$ & 159.030 & $\mathrm{C}_{6} \mathrm{H}_{7} \mathrm{O}_{5}^{-}$ & $\mathrm{M}-\left(\mathrm{H}_{2} \mathrm{O}\right)-3 \mathrm{H}$ \\
$\ldots$ & 177.040 & $\mathrm{C}_{6} \mathrm{H}_{9} \mathrm{O}_{6}^{-}$ & $\mathrm{M}-3 \mathrm{H}$ \\
$\ldots$ & 179.056 & $\mathrm{C}_{6} \mathrm{H}_{11} \mathrm{O}_{6}^{-}$ & $\mathrm{M}-\mathrm{H}$ \\
\hline & 359.120 & $\mathrm{C}_{12} \mathrm{H}_{23} \mathrm{O}_{12}^{-}$ & $2 \mathrm{M}-\mathrm{H}$ \\
\hline
\end{tabular}

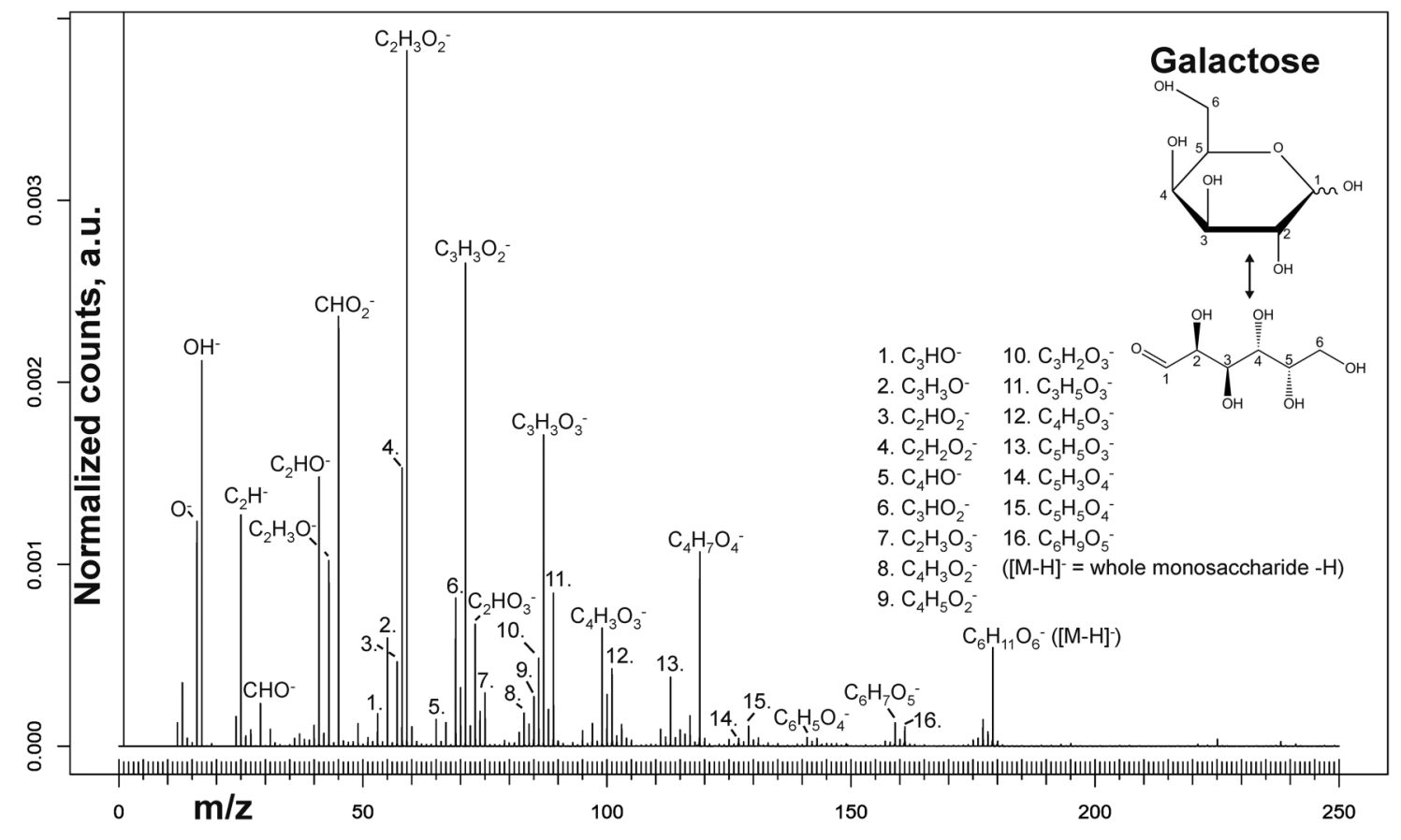

\begin{tabular}{rc}
\hline Accession \# & $01577-01$ \\
\hline Host Material: \\
Technique: \\
$\begin{array}{r}\text { Mass Range: } \\
\text { Secondary Source Polarity: }\end{array}$ & Silicon wafer \\
SIMS & Negative \\
Species Used for Mass Calibration: & $0-250 \mathrm{Da}$ \\
Primary lon Dose: & $\mathrm{C}^{-}, \mathrm{CH}^{-}, \mathrm{CH}_{2}^{-}, \mathrm{CH}_{3} \mathrm{O}^{-}, \mathrm{C}_{4} \mathrm{H}_{3}{ }^{-}, \mathrm{C}_{3} \mathrm{HO}^{-}, \mathrm{C}_{2} \mathrm{HO}_{2}{ }^{-}$ \\
$1.01 \times 10^{12} \mathrm{~cm}^{-2}$ \\
$17.2 \mathrm{~ns}$ \\
Primary lon Pulse Width: & $0.00079 \mathrm{nA}$ \\
Pulsed Beam Current: & D-Galactose is found in a wide range of glycoproteins and \\
Biological significance: & glycolipids, and galactose-based polysaccharides occur in certain \\
& type of hemicellulose. The milk sugar lactose is a disaccharide \\
& composed of one D-galactose and one D-glucose unit \\
\hline
\end{tabular}




\begin{tabular}{lccc}
\hline & & 2-DEOXY-D-GALACTOSE & \\
\hline Spectrum ID \# & Mass (Da) & Species & Peak Assignment \\
\hline 01578 & 71.014 & $\mathrm{C}_{3} \mathrm{H}_{3} \mathrm{O}_{2}^{-}$ & $\mathrm{C} 1$ to $\mathrm{C} 3$ fragment of $\mathrm{M}-2 \mathrm{H}$ \\
$\ldots$ & 85.030 & $\mathrm{C}_{4} \mathrm{H}_{5} \mathrm{O}_{2}^{-}$ & $\mathrm{C} 1$ to $\mathrm{C} 4$ fragment of $\mathrm{M}-\left(\mathrm{H}_{2} \mathrm{O}\right)$ \\
$\ldots$ & 99.009 & $\mathrm{C}_{4} \mathrm{H}_{3} \mathrm{O}_{3}^{-}$ & $\mathrm{C} 1$ to C4 fragment of $\mathrm{M}-4 \mathrm{H}$ \\
$\ldots$ & 119.035 & $\mathrm{C}_{4} \mathrm{H}_{7} \mathrm{O}_{4}^{-}$ & $\mathrm{C} 3$ to C6 fragment of $\mathrm{M}-2 \mathrm{H}$ \\
$\ldots$ & 125.024 & $\mathrm{C}_{6} \mathrm{H}_{5} \mathrm{O}_{3}^{-}$ & $\mathrm{M}-2\left(\mathrm{H}_{2} \mathrm{O}\right)-3 \mathrm{H}$ \\
$\ldots$ & 163.061 & $\mathrm{C}_{6} \mathrm{H}_{11} \mathrm{O}_{5}^{-}$ & $\mathrm{M}-\mathrm{H}$ \\
$\ldots$ & 327.130 & $\mathrm{C}_{12} \mathrm{H}_{23} \mathrm{O}_{10}$ & $2 \mathrm{M}-\mathrm{H}$ \\
\hline
\end{tabular}

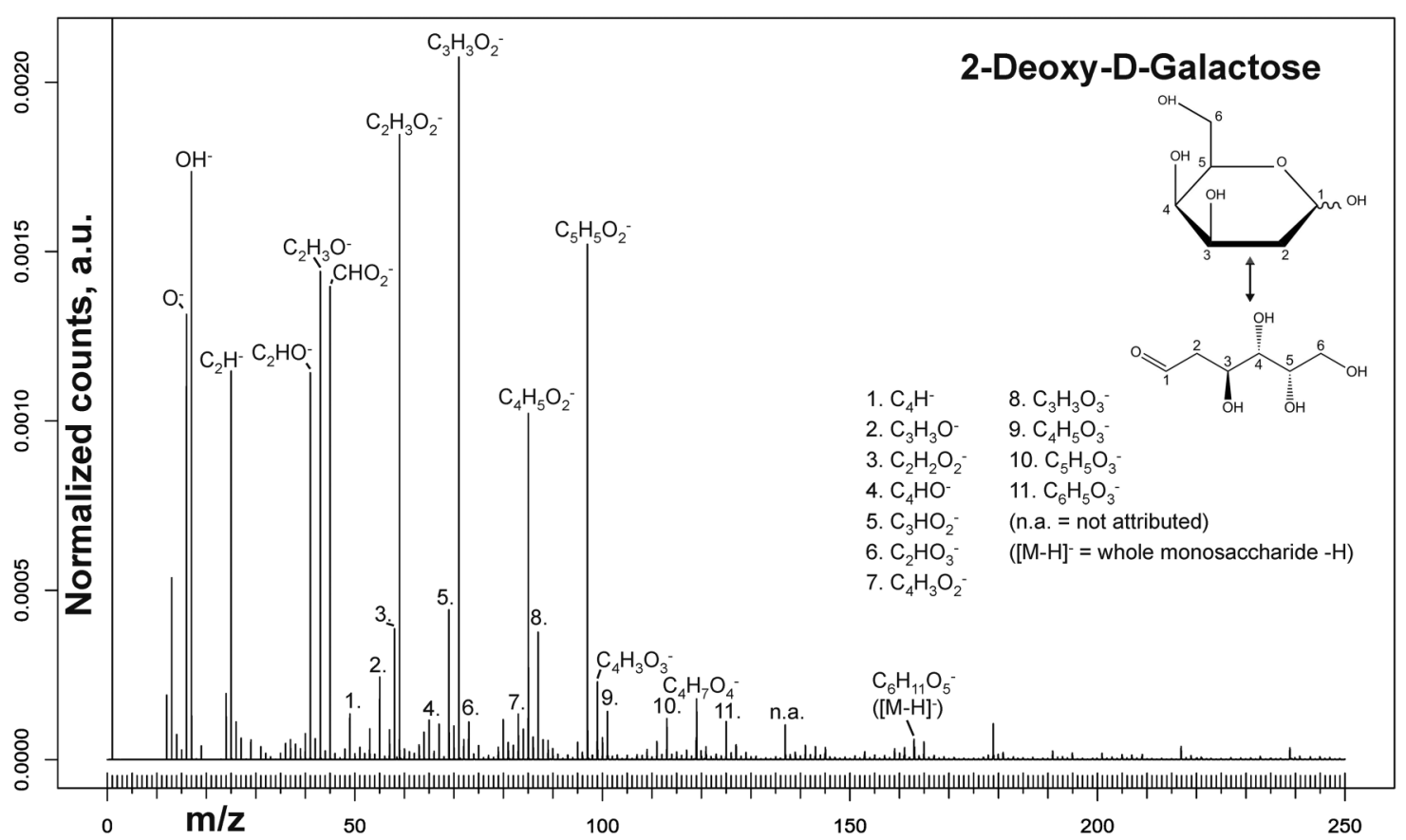

\begin{tabular}{rc}
\hline Accession \# & $01578-01$ \\
\hline Host Material: & Tilicon wafer \\
Technique: & SIMS \\
Secondary Source Polarity: & Negative \\
Mass Range: & $0-250 \mathrm{Da}$ \\
Species Used for Mass Calibration: & $\mathrm{C}^{-}, \mathrm{CH}^{-}, \mathrm{CH}_{2}^{-}, \mathrm{CH}_{3} \mathrm{O}^{-}, \mathrm{C}_{4} \mathrm{H}_{3}^{-}, \mathrm{C}_{3} \mathrm{HO}^{-}, \mathrm{C}_{2} \mathrm{HO}_{2}^{-}$ \\
Primary lon Dose: & $9.46 \times 10^{11} \mathrm{~cm}^{-2}$ \\
Primary lon Pulse Width: & $17.2 \mathrm{~ns}$ \\
Pulsed Beam Current: & $0.00074 \mathrm{nA}$ \\
Biological significance: & 2-Deoxy-D-galactose is not abundant in nature but of scientific \\
& relevance as a known inhibitor of membrane glycoprotein synthesis \\
\hline
\end{tabular}




\section{D-GALACTURONIC ACID}

\begin{tabular}{|c|c|c|c|}
\hline Spectrum ID \# & Mass (Da) & Species & Peak Assignment \\
\hline 01579 & 71.014 & $\mathrm{C}_{3} \mathrm{H}_{3} \mathrm{O}_{2}^{-}$ & $\mathrm{C} 1$ to $\mathrm{C} 3$ fragment of $\mathrm{M}-\left(\mathrm{H}_{2} \mathrm{O}\right)$ \\
\hline$\ldots$ & 72.993 & $\mathrm{C}_{2} \mathrm{HO}_{3}^{-}$ & $\mathrm{C} 5$ to $\mathrm{C} 6$ fragment of $\mathrm{M}-2 \mathrm{H}$ \\
\hline$\ldots$ & 75.009 & $\mathrm{C}_{2} \mathrm{H}_{3} \mathrm{O}_{3}^{-}$ & C5 to C6 fragment of M \\
\hline$\ldots$ & 87.009 & $\mathrm{C}_{3} \mathrm{H}_{3} \mathrm{O}_{3}^{-}$ & $\mathrm{C} 1$ to $\mathrm{C} 3$ fragment of $\mathrm{M}-2 \mathrm{H}$ \\
\hline$\ldots$ & 99.009 & $\mathrm{C}_{4} \mathrm{H}_{3} \mathrm{O}_{3}^{-}$ & $\mathrm{C} 1$ to $\mathrm{C} 4$ fragment of $\mathrm{M}-\left(\mathrm{H}_{2} \mathrm{O}\right)-2 \mathrm{H}$ \\
\hline$\ldots$ & 103.004 & $\mathrm{C}_{3} \mathrm{H}_{3} \mathrm{O}_{4}^{-}$ & $\mathrm{C} 4$ to $\mathrm{C} 6$ fragment of $\mathrm{M}-2 \mathrm{H}$ \\
\hline$\ldots$ & 115.004 & $\mathrm{C}_{4} \mathrm{H}_{3} \mathrm{O}_{4}^{-}$ & $\mathrm{C} 3$ to $\mathrm{C} 6$ fragment of $\mathrm{M}-\left(\mathrm{H}_{2} \mathrm{O}\right)-2 \mathrm{H}$ \\
\hline$\ldots$ & 133.014 & $\mathrm{C}_{4} \mathrm{H}_{5} \mathrm{O}_{5}^{-}$ & $\mathrm{C} 3$ to $\mathrm{C} 6$ fragment of $\mathrm{M}-2 \mathrm{H}$ \\
\hline$\ldots$ & 193.020 & $\mathrm{C}_{6} \mathrm{H}_{7} \mathrm{O}_{7}^{-}$ & $M-3 H$ \\
\hline$\ldots$ & 193.035 & $\mathrm{C}_{6} \mathrm{H}_{9} \mathrm{O}_{7}^{-}$ & $\mathrm{M}-\mathrm{H}$ \\
\hline$\ldots$ & 387.078 & $\mathrm{C}_{12} \mathrm{H}_{19} \mathrm{O}_{14}^{-}$ & $2 \mathrm{M}-\mathrm{H}$ \\
\hline
\end{tabular}

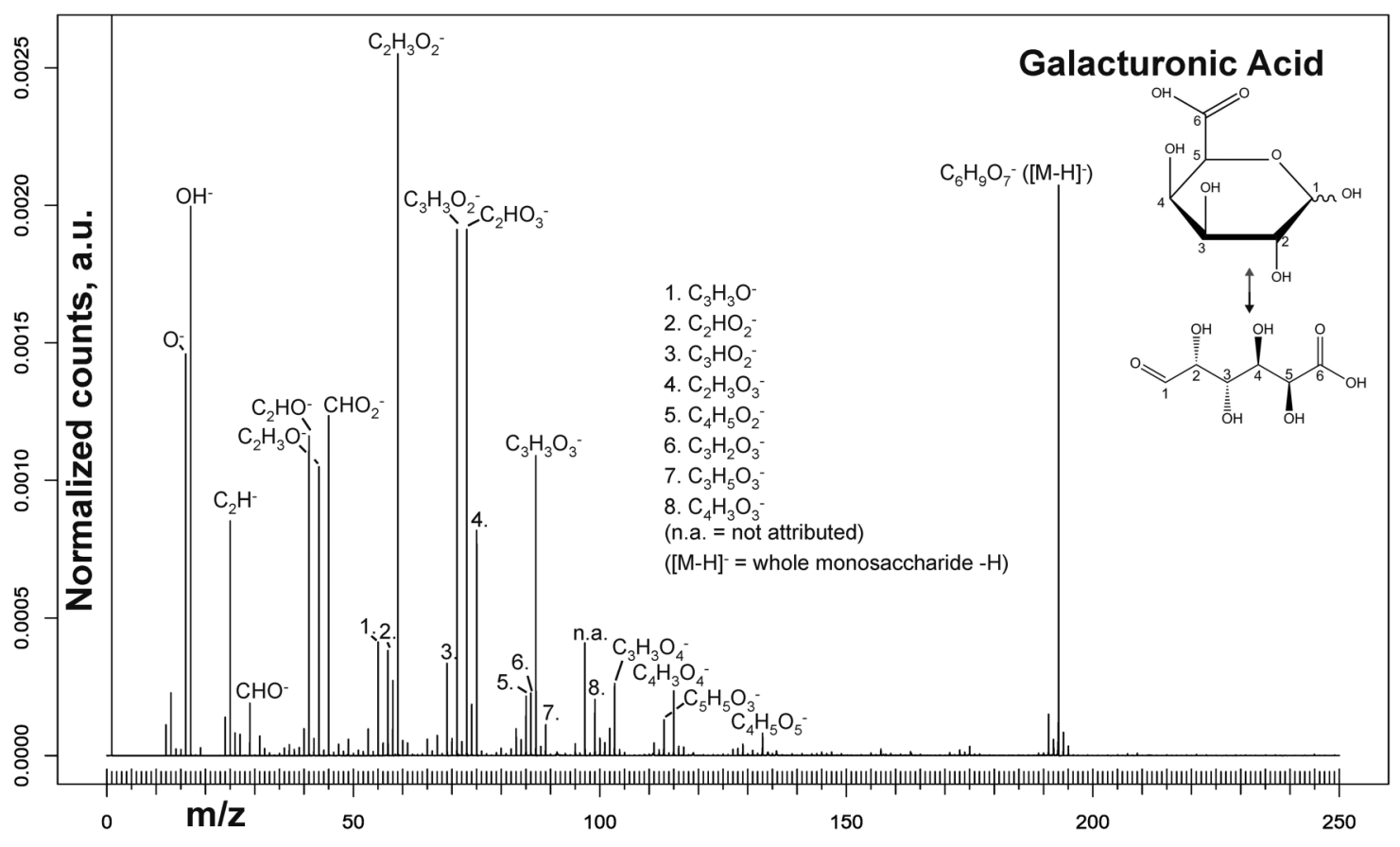

\begin{tabular}{rc}
\hline Accession \# & $01579-01$ \\
\hline Host Material: \\
Technique: & Silicon wafer \\
Mass Range: & SIMS \\
Negative \\
Secondary Source Polarity: & $0-250 \mathrm{Da}$ \\
Species Used for Mass & $\mathrm{C}^{-}, \mathrm{CH}^{-}, \mathrm{CH}_{2}^{-}, \mathrm{CH}_{3} \mathrm{O}^{-}, \mathrm{C}_{4} \mathrm{H}_{3}^{-}, \mathrm{C}_{3} \mathrm{HO}^{-}, \mathrm{C}_{2} \mathrm{HO}_{2}{ }^{-}$ \\
Calibration: & $9.46 \times 10^{11} \mathrm{~cm}^{-2}$ \\
Primary lon Dose: & $17.2 \mathrm{~ns}$ \\
Primary lon Pulse Width: & $0.00074 \mathrm{nA}$ \\
Pulsed Beam Current: & D-Galacturonic acid is the uronic acid of D-galactose and is, among \\
Biological significance: & others, the main component of the plant cell wall \\
& heteropolysaccharide pectin \\
\hline
\end{tabular}




\begin{tabular}{lccc}
\hline & & D-GLUCOSE & \\
\hline Spectrum ID \# & Mass (Da) & Species & Peak Assignment \\
\hline 01580 & 71.014 & $\mathrm{C}_{3} \mathrm{H}_{3} \mathrm{O}_{2}^{-}$ & $\mathrm{C} 1$ to $\mathrm{C}$ fragment of $\mathrm{M}-\mathrm{H}_{2} \mathrm{O}$ \\
$\ldots$ & 87.009 & $\mathrm{C}_{3} \mathrm{H}_{3} \mathrm{O}_{3}^{-}$ & $\mathrm{C} 1$ to $\mathrm{C}$ fragment of $\mathrm{M}-2 \mathrm{H}$ \\
$\ldots$ & 99.009 & $\mathrm{C}_{4} \mathrm{H}_{3} \mathrm{O}_{3}^{-}$ & $\mathrm{C} 1$ to $\mathrm{C}$ fragment of $\mathrm{M}-\mathrm{H}_{2} \mathrm{O}-2 \mathrm{H}$ \\
$\ldots$ & 119.035 & $\mathrm{C}_{4} \mathrm{H}_{7} \mathrm{O}_{4}^{-}$ & $\mathrm{C}$ to $\mathrm{C}$ fragment of $\mathrm{M}-2 \mathrm{H}$ \\
$\ldots$ & 141.019 & $\mathrm{C}_{6} \mathrm{H}_{5} \mathrm{O}_{4}^{-}$ & $\mathrm{M}-2\left(\mathrm{H}_{2} \mathrm{O}\right)-3 \mathrm{H}$ \\
$\ldots$ & 159.030 & $\mathrm{C}_{6} \mathrm{H}_{7} \mathrm{O}_{5}^{-}$ & $\mathrm{M}-\left(\mathrm{H}_{2} \mathrm{O}\right)-3 \mathrm{H}$ \\
$\ldots$ & 177.040 & $\mathrm{C}_{6} \mathrm{H}_{9} \mathrm{O}_{6}^{-}$ & $\mathrm{M}-3 \mathrm{H}$ \\
$\ldots$ & 179.056 & $\mathrm{C}_{6} \mathrm{H}_{11} \mathrm{O}_{6}^{-}$ & $\mathrm{M}-\mathrm{H}$ \\
\hline & 359.120 & $\mathrm{C}_{12} \mathrm{H}_{23} \mathrm{O}_{12}^{-}$ & $2 \mathrm{M}-\mathrm{H}$ \\
\hline
\end{tabular}

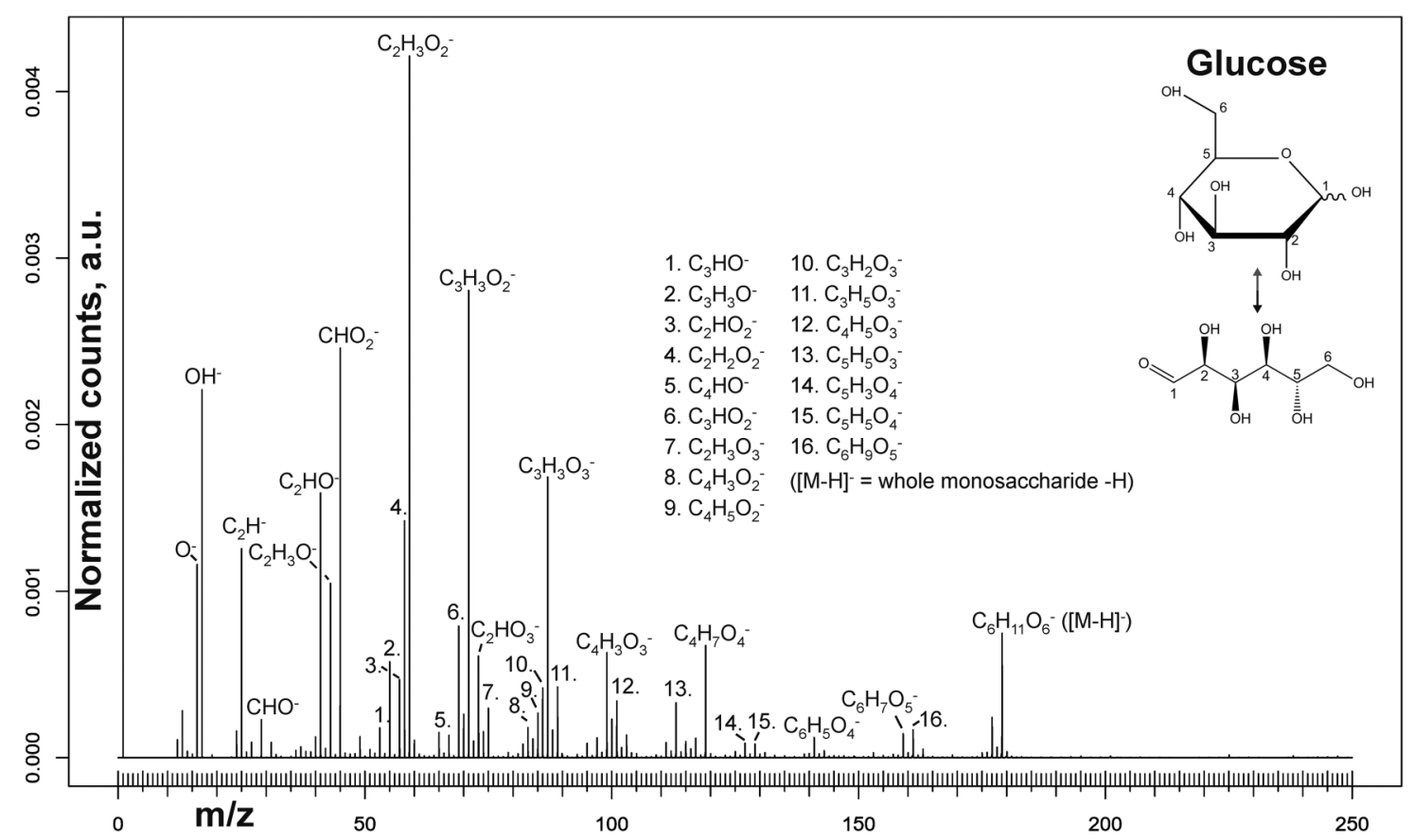

\begin{tabular}{|c|c|}
\hline Accession \# & 01580-01 \\
\hline Host Material: & Silicon wafer \\
\hline Technique: & SIMS \\
\hline Secondary Source Polarity: & Negative \\
\hline Mass Range: & $0-250 \mathrm{Da}$ \\
\hline Species Used for Mass Calibration: & $\mathrm{C}^{-}, \mathrm{CH}^{-}, \mathrm{CH}_{2}^{-}, \mathrm{CH}_{3} \mathrm{O}^{-}, \mathrm{C}_{4} \mathrm{H}_{3}^{-}, \mathrm{C}_{3} \mathrm{HO}^{-}, \mathrm{C}_{2} \mathrm{HO}_{2}^{-}$ \\
\hline Primary lon Dose: & $1.01 \times 10^{12} \mathrm{~cm}^{-2}$ \\
\hline Primary lon Pulse Width: & $17.2 \mathrm{~ns}$ \\
\hline Pulsed Beam Current: & $0.00079 \mathrm{nA}$ \\
\hline Biological significance: & $\begin{array}{c}\text { Glucose is essential for energy storage, energy transport, and cell } \\
\text { metabolism in animals, plants, and microorganisms. Glucose is the } \\
\text { monomer unit of the polysaccharides starch and glycogen, polymers } \\
\text { acting as energy storage in plants and animals, respectively, and the } \\
\text { polysaccharide cellulose, which is the main plant cell wall } \\
\text { component. Glucose is also part of many di- and oligosaccharides, } \\
\text { e.g., sucrose, lactose, and trehalose }\end{array}$ \\
\hline
\end{tabular}




\begin{tabular}{|c|c|c|c|}
\hline \multicolumn{4}{|c|}{ D-GLUCURONIC ACID } \\
\hline Spectrum ID \# & Mass (Da) & Species & Peak Assignment \\
\hline 01581 & 71.014 & $\mathrm{C}_{3} \mathrm{H}_{3} \mathrm{O}_{2}^{-}$ & $\mathrm{C} 1$ to $\mathrm{C} 3$ fragment of $\mathrm{M}-\left(\mathrm{H}_{2} \mathrm{O}\right)$ \\
\hline$\ldots$ & 72.993 & $\mathrm{C}_{2} \mathrm{HO}_{3}^{-}$ & $\mathrm{C} 5$ to $\mathrm{C} 6$ fragment of $\mathrm{M}-2 \mathrm{H}$ \\
\hline$\ldots$ & 75.009 & $\mathrm{C}_{2} \mathrm{H}_{3} \mathrm{O}_{3}^{-}$ & C5 to C6 fragment of M \\
\hline$\ldots$ & 87.009 & $\mathrm{C}_{3} \mathrm{H}_{3} \mathrm{O}_{3}^{-}$ & $\mathrm{C} 1$ to $\mathrm{C} 3$ fragment of $\mathrm{M}-2 \mathrm{H}$ \\
\hline$\ldots$ & 99.009 & $\mathrm{C}_{4} \mathrm{H}_{3} \mathrm{O}_{3}^{-}$ & $\mathrm{C} 1$ to $\mathrm{C} 4$ fragment of $\mathrm{M}-\left(\mathrm{H}_{2} \mathrm{O}\right)-2 \mathrm{H}$ \\
\hline$\ldots$ & 103.004 & $\mathrm{C}_{3} \mathrm{H}_{3} \mathrm{O}_{4}^{-}$ & $\mathrm{C} 4$ to $\mathrm{C} 6$ fragment of $\mathrm{M}-2 \mathrm{H}$ \\
\hline$\ldots$ & 115.004 & $\mathrm{C}_{4} \mathrm{H}_{3} \mathrm{O}_{4}^{-}$ & $\mathrm{C} 3$ to $\mathrm{C} 6$ fragment of $\mathrm{M}-\left(\mathrm{H}_{2} \mathrm{O}\right)-2 \mathrm{H}$ \\
\hline$\ldots$ & 133.014 & $\mathrm{C}_{4} \mathrm{H}_{5} \mathrm{O}_{5}^{-}$ & $\mathrm{C} 3$ to $\mathrm{C} 6$ fragment of $\mathrm{M}-2 \mathrm{H}$ \\
\hline$\ldots$ & 193.020 & $\mathrm{C}_{6} \mathrm{H}_{7} \mathrm{O}_{7}^{-}$ & $M-3 H$ \\
\hline$\ldots$ & 193.035 & $\mathrm{C}_{6} \mathrm{H}_{9} \mathrm{O}_{7}^{-}$ & $\mathrm{M}-\mathrm{H}$ \\
\hline$\ldots$ & 387.078 & $\mathrm{C}_{12} \mathrm{H}_{19} \mathrm{O}_{14}^{-}$ & $2 \mathrm{M}-\mathrm{H}$ \\
\hline
\end{tabular}

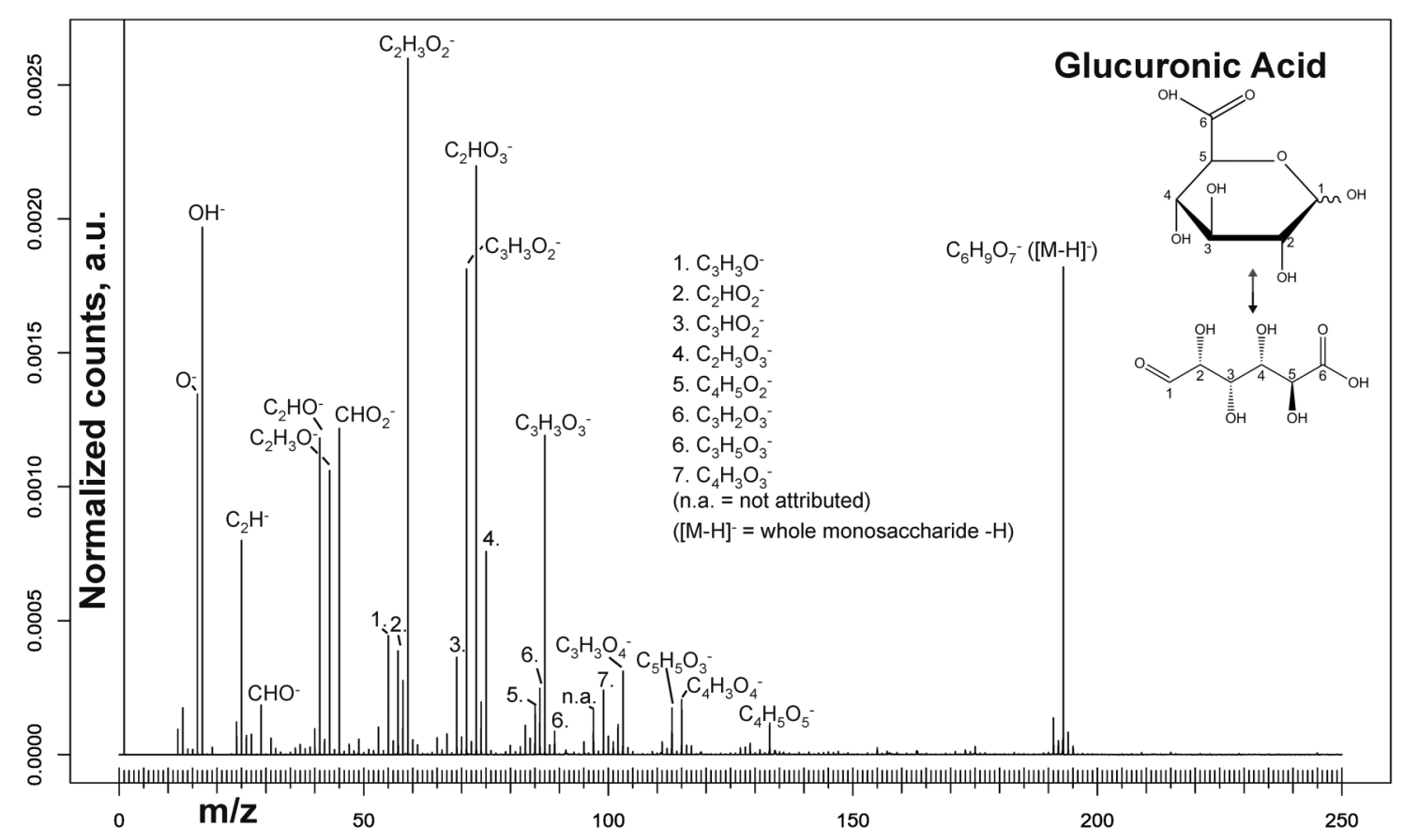

\begin{tabular}{rc}
\hline Accession \# & $01581-01$ \\
\hline Host Material: & Silicon wafer \\
Technique: & SIMS \\
Mass Range: & Negative \\
Secondary Source Polarity: & $0-250 \mathrm{Da}$ \\
Species Used for Mass & \\
Calibration: & $\mathrm{C}^{-}, \mathrm{CH}^{-}, \mathrm{CH}_{2}^{-}, \mathrm{CH}_{3} \mathrm{O}^{-}, \mathrm{C}_{4} \mathrm{H}_{3}^{-}, \mathrm{C}_{3} \mathrm{HO}^{-}, \mathrm{C}_{2} \mathrm{HO}_{2}^{-}$ \\
Primary lon Dose: & $9.46 \times 10^{11} \mathrm{~cm}^{-2}$ \\
Primary lon Pulse Width: & $17.2 \mathrm{~ns}$ \\
Pulsed Beam Current: & $0.00074 \mathrm{nA}$ \\
Biological significance: & Glucuronic acid is the uronic acid of D-glucose and plays a major role in \\
& the phase II detoxification processes in the liver, where, in an \\
& enzyme-driven reaction, it is bound to toxins to facilitate their excretion \\
\hline
\end{tabular}




\begin{tabular}{lccc}
\hline & & D-MANNOSE & \\
\hline Spectrum ID \# & Mass (Da) & Species & Peak Assignment \\
\hline 01582 & 71.014 & $\mathrm{C}_{3} \mathrm{H}_{3} \mathrm{O}_{2}^{-}$ & $\mathrm{C} 1$ to $\mathrm{C}$ fragment of $\mathrm{M}-\mathrm{H}_{2} \mathrm{O}$ \\
$\ldots$ & 87.009 & $\mathrm{C}_{3} \mathrm{H}_{3} \mathrm{O}_{3}^{-}$ & $\mathrm{C} 1$ to $\mathrm{C}$ fragment of $\mathrm{M}-2 \mathrm{H}$ \\
$\ldots$ & 99.009 & $\mathrm{C}_{4} \mathrm{H}_{3} \mathrm{O}_{3}^{-}$ & $\mathrm{C} 1$ to $\mathrm{C}$ fragment of $\mathrm{M}-\mathrm{H}_{2} \mathrm{O}-2 \mathrm{H}$ \\
$\ldots$ & 119.035 & $\mathrm{C}_{4} \mathrm{H}_{7} \mathrm{O}_{4}^{-}$ & $\mathrm{C}$ to $\mathrm{C}$ fragment of $\mathrm{M}-2 \mathrm{H}$ \\
$\ldots$ & 141.019 & $\mathrm{C}_{6} \mathrm{H}_{5} \mathrm{O}_{4}^{-}$ & $\mathrm{M}-2\left(\mathrm{H}_{2} \mathrm{O}\right)-3 \mathrm{H}$ \\
$\ldots$ & 159.030 & $\mathrm{C}_{6} \mathrm{H}_{7} \mathrm{O}_{5}^{-}$ & $\mathrm{M}-\left(\mathrm{H}_{2} \mathrm{O}\right)-3 \mathrm{H}$ \\
$\ldots$ & 177.040 & $\mathrm{C}_{6} \mathrm{H}_{9} \mathrm{O}_{6}^{-}$ & $\mathrm{M}-3 \mathrm{H}$ \\
$\ldots$ & 179.056 & $\mathrm{C}_{6} \mathrm{H}_{11} \mathrm{O}_{6}^{-}$ & $\mathrm{M}-\mathrm{H}$ \\
\hline & 359.120 & $\mathrm{C}_{12} \mathrm{H}_{23} \mathrm{O}_{12}^{-}$ & $2 \mathrm{M}-\mathrm{H}$ \\
\hline
\end{tabular}

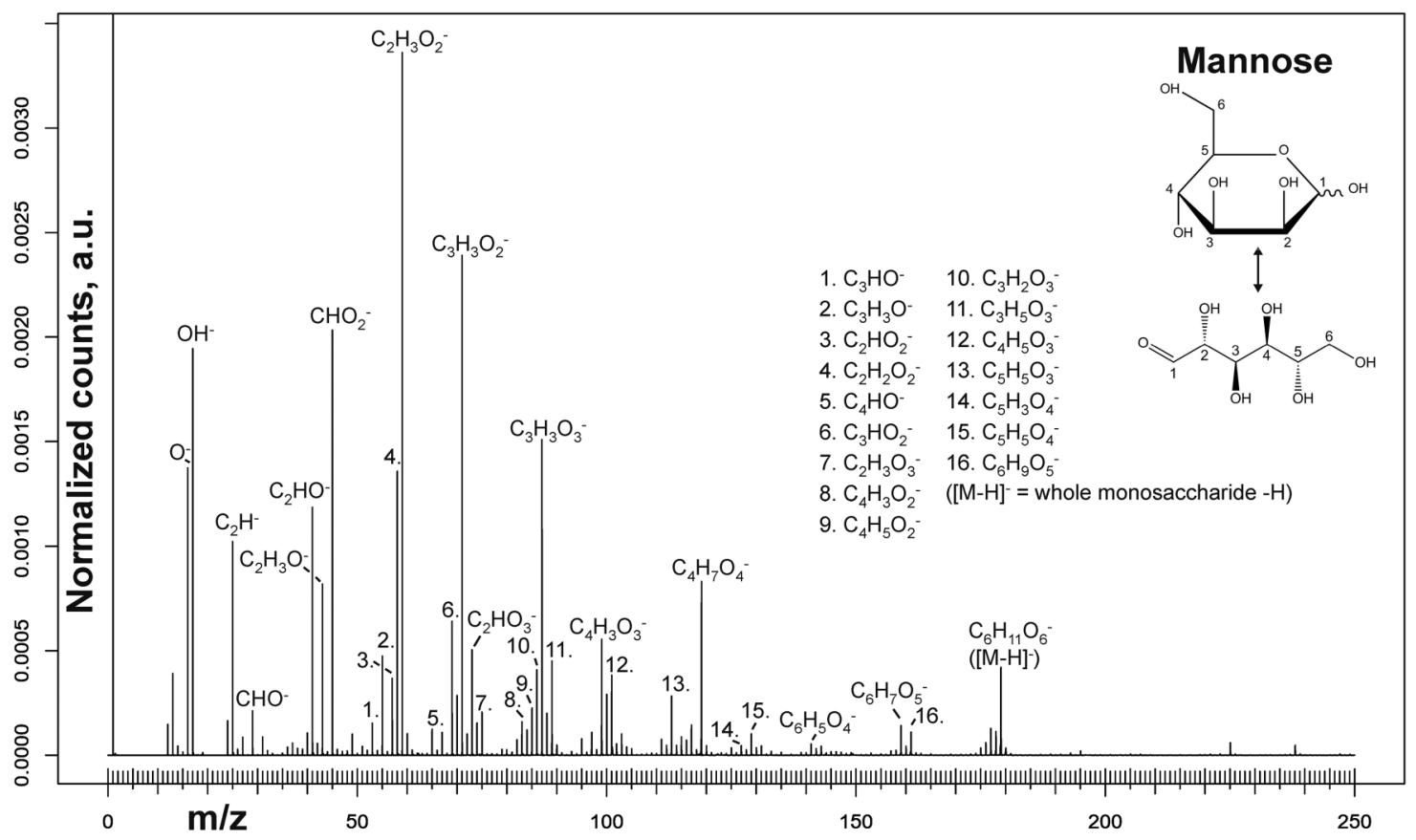

\begin{tabular}{|c|c|}
\hline Accession \# & 01582-01 \\
\hline Host Material: & Silicon wafer \\
\hline ■ Technique: & SIMS \\
\hline Secondary Source Polarity: & Negative \\
\hline Mass Range: & $0-250 \mathrm{Da}$ \\
\hline $\begin{array}{r}\text { Species Used for Mass } \\
\text { Calibration: }\end{array}$ & $\mathrm{C}^{-}, \mathrm{CH}^{-}, \mathrm{CH}_{2}^{-}, \mathrm{CH}_{3} \mathrm{O}^{-}, \mathrm{C}_{4} \mathrm{H}_{3}^{-}, \mathrm{C}_{3} \mathrm{HO}^{-}, \mathrm{C}_{2} \mathrm{HO}_{2}^{-}$ \\
\hline Primary lon Dose: & $9.71 \times 10^{11} \mathrm{~cm}^{-2}$ \\
\hline Primary Ion Pulse Width: & $17.2 \mathrm{~ns}$ \\
\hline Pulsed Beam Current: & $0.00076 \mathrm{nA}$ \\
\hline Biological significance: & $\begin{array}{l}\text { D-Mannose is part of the glycosylation process of certain } \\
\text { glycoproteins. During the C-mannosylation process, a mannose } \\
\text { monosaccharide attaches by covalent bond formation to tryptophan }\end{array}$ \\
\hline
\end{tabular}




\begin{tabular}{lccc}
\hline & & MYOINOSITOL & \\
\hline Spectrum ID \# & Mass (Da) & Species & Peak Assignment \\
\hline 01583 & 71.014 & $\mathrm{C}_{3} \mathrm{H}_{3} \mathrm{O}_{2}^{-}$ & $\mathrm{C} 1$ to $\mathrm{C}$ fragment of $\mathrm{M}-\mathrm{H}_{2} \mathrm{O}$ \\
$\ldots$ & 87.009 & $\mathrm{C}_{3} \mathrm{H}_{3} \mathrm{O}_{3}^{-}$ & $\mathrm{C} 1$ to $\mathrm{C}$ fragment of $\mathrm{M}-2 \mathrm{H}$ \\
$\ldots$ & 99.009 & $\mathrm{C}_{4} \mathrm{H}_{3} \mathrm{O}_{3}^{-}$ & $\mathrm{C} 1$ to $\mathrm{C} 4$ fragment of $\mathrm{M}-\mathrm{H}_{2} \mathrm{O}-2 \mathrm{H}$ \\
$\ldots$ & 113.024 & $\mathrm{C}_{5} \mathrm{H}_{5} \mathrm{O}_{3}^{-}$ & - \\
$\ldots$ & 125.024 & $\mathrm{C}_{6} \mathrm{H}_{5} \mathrm{O}_{3}^{-}$ & $\mathrm{M}-3\left(\mathrm{H}_{2} \mathrm{O}\right)-\mathrm{H}$ \\
$\ldots$ & 142.027 & $\mathrm{C}_{6} \mathrm{H}_{6} \mathrm{O}_{4}^{-}$ & $\mathrm{M}-2\left(\mathrm{H}_{2} \mathrm{O}\right)-2 \mathrm{H}$ \\
$\ldots$ & 159.030 & $\mathrm{C}_{6} \mathrm{H}_{7} \mathrm{O}_{5}^{-}$ & $\mathrm{M}-\left(\mathrm{H}_{2} \mathrm{O}\right)-3 \mathrm{H}$ \\
$\ldots$ & 179.056 & $\mathrm{C}_{6} \mathrm{H}_{11} \mathrm{O}_{6}^{-}$ & $\mathrm{M}-\mathrm{H}$ \\
$\ldots$ & 359.120 & $\mathrm{C}_{12} \mathrm{H}_{23} \mathrm{O}_{12}^{-}$ & $2 \mathrm{M}-\mathrm{H}$ \\
\hline
\end{tabular}

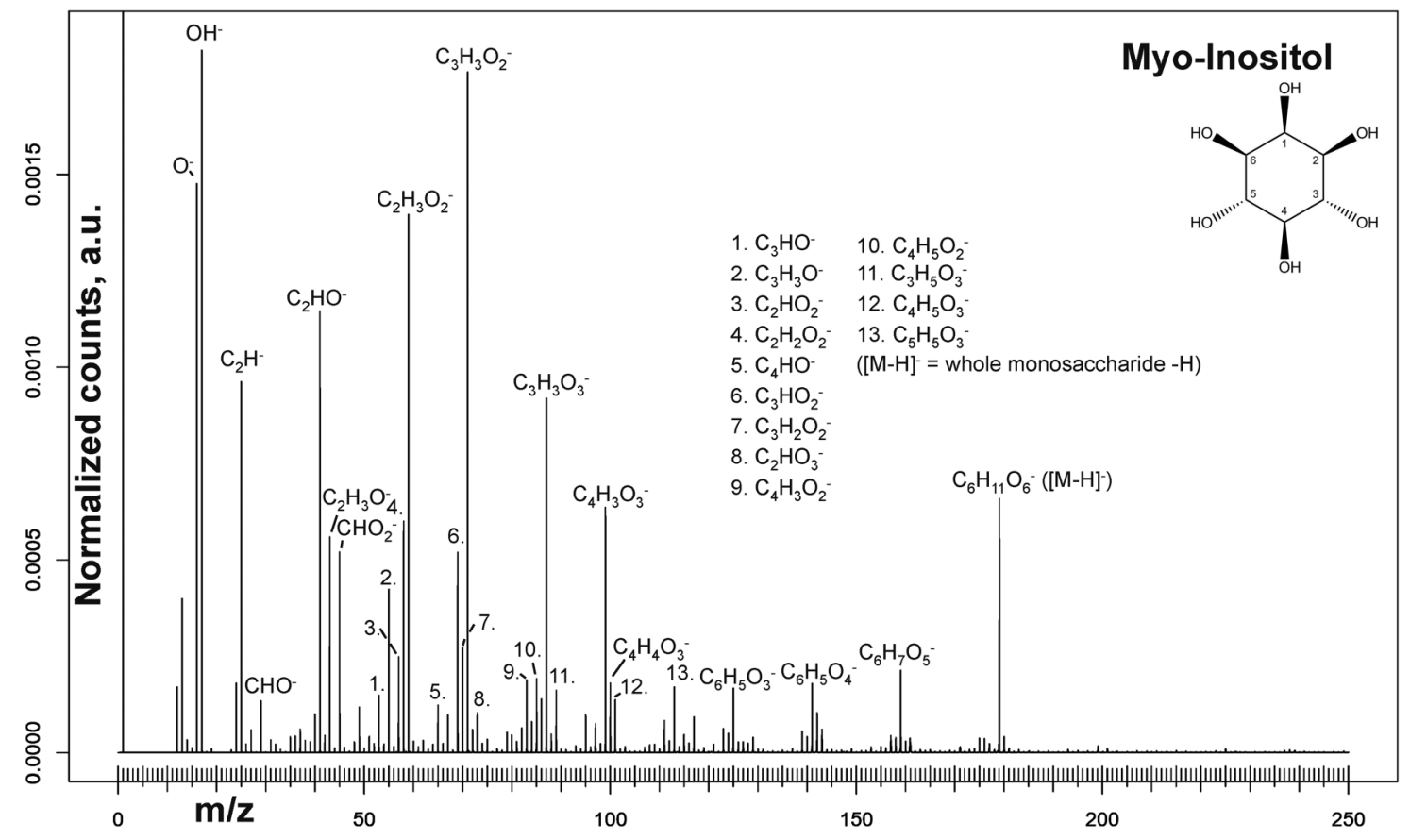

\begin{tabular}{|c|c|}
\hline Accession \# & 01583-01 \\
\hline Host Material: & Silicon wafer \\
\hline 口 Technique: & SIMS \\
\hline Secondary Source Polarity: & Negative \\
\hline Mass Range: & $0-250 \mathrm{Da}$ \\
\hline $\begin{array}{r}\text { Species Used for Mass } \\
\text { Calibration: }\end{array}$ & $\mathrm{C}^{-}, \mathrm{CH}^{-}, \mathrm{CH}_{2}^{-}, \mathrm{CH}_{3} \mathrm{O}^{-}, \mathrm{C}_{4} \mathrm{H}_{3}^{-}, \mathrm{C}_{3} \mathrm{HO}^{-}, \mathrm{C}_{2} \mathrm{HO}_{2}^{-}$ \\
\hline Primary lon Dose: & $9.46 \times 10^{11} \mathrm{~cm}^{-2}$ \\
\hline Primary Ion Pulse Width: & $17.2 \mathrm{~ns}$ \\
\hline Pulsed Beam Current: & $0.00074 \mathrm{nA}$ \\
\hline Biological significance: & $\begin{array}{l}\text { Myoinositol derivatives serve as a } \mathrm{Ca}^{2+} \text { secondary messenger; } \\
\text { myoinositol is also the basis for the phosphate storing molecule } \\
\text { phytic acid in certain plants, as well as the component of a wide } \\
\text { range of phospholipids }\end{array}$ \\
\hline
\end{tabular}




\begin{tabular}{lccc}
\hline & & L-RHAMNOSE & \\
\hline Spectrum ID \# & Mass (Da) & Species & Peak Assignment \\
\hline 01584 & 71.014 & $\mathrm{C}_{3} \mathrm{H}_{3} \mathrm{O}_{2}{ }^{-}$ & $\mathrm{C} 1$ to $\mathrm{C} 3$ fragment of $\mathrm{M}-2 \mathrm{H}$ \\
$\ldots$ & 85.030 & $\mathrm{C}_{4} \mathrm{H}_{5} \mathrm{O}_{2}^{-}$ & $\mathrm{C} 1$ to $\mathrm{C} 4$ fragment of $\mathrm{M}-\left(\mathrm{H}_{2} \mathrm{O}\right)$ \\
$\ldots$ & 87.009 & $\mathrm{C}_{3} \mathrm{H}_{3} \mathrm{O}_{3}^{-}$ & Pyruvate \\
$\ldots$ & 99.009 & $\mathrm{C}_{4} \mathrm{H}_{3} \mathrm{O}_{3}^{-}$ & $\mathrm{C} 1$ to C4 fragment of $\mathrm{M}-4 \mathrm{H}$ \\
$\ldots$ & 103.040 & $\mathrm{C}_{4} \mathrm{H}_{7} \mathrm{O}_{3}^{-}$ & $\mathrm{C} 3$ to C6 fragment of $\mathrm{M}-2 \mathrm{H}$ \\
$\ldots$ & 119.035 & $\mathrm{C}_{4} \mathrm{H}_{7} \mathrm{O}_{4}^{-}$ & $\mathrm{C} 1$ to C4 fragment of M \\
$\ldots$ & 127.004 & $\mathrm{C}_{5} \mathrm{H}_{3} \mathrm{O}_{4}^{-}$ & $\ldots$ \\
$\ldots$ & 141.019 & $\mathrm{C}_{6} \mathrm{H}_{9} \mathrm{O}_{4}^{-}$ & $\mathrm{M}-\mathrm{H}-\left(\mathrm{H}_{2} \mathrm{O}\right)$ \\
$\ldots$ & 163.061 & $\mathrm{C}_{6} \mathrm{H}_{11} \mathrm{O}_{5}^{-}$ & $\mathrm{M}-\mathrm{H}$ \\
\hline & 327.130 & $\mathrm{C}_{12} \mathrm{H}_{23} \mathrm{O}_{10}$ & $2 \mathrm{M}-\mathrm{H}$ \\
\hline
\end{tabular}

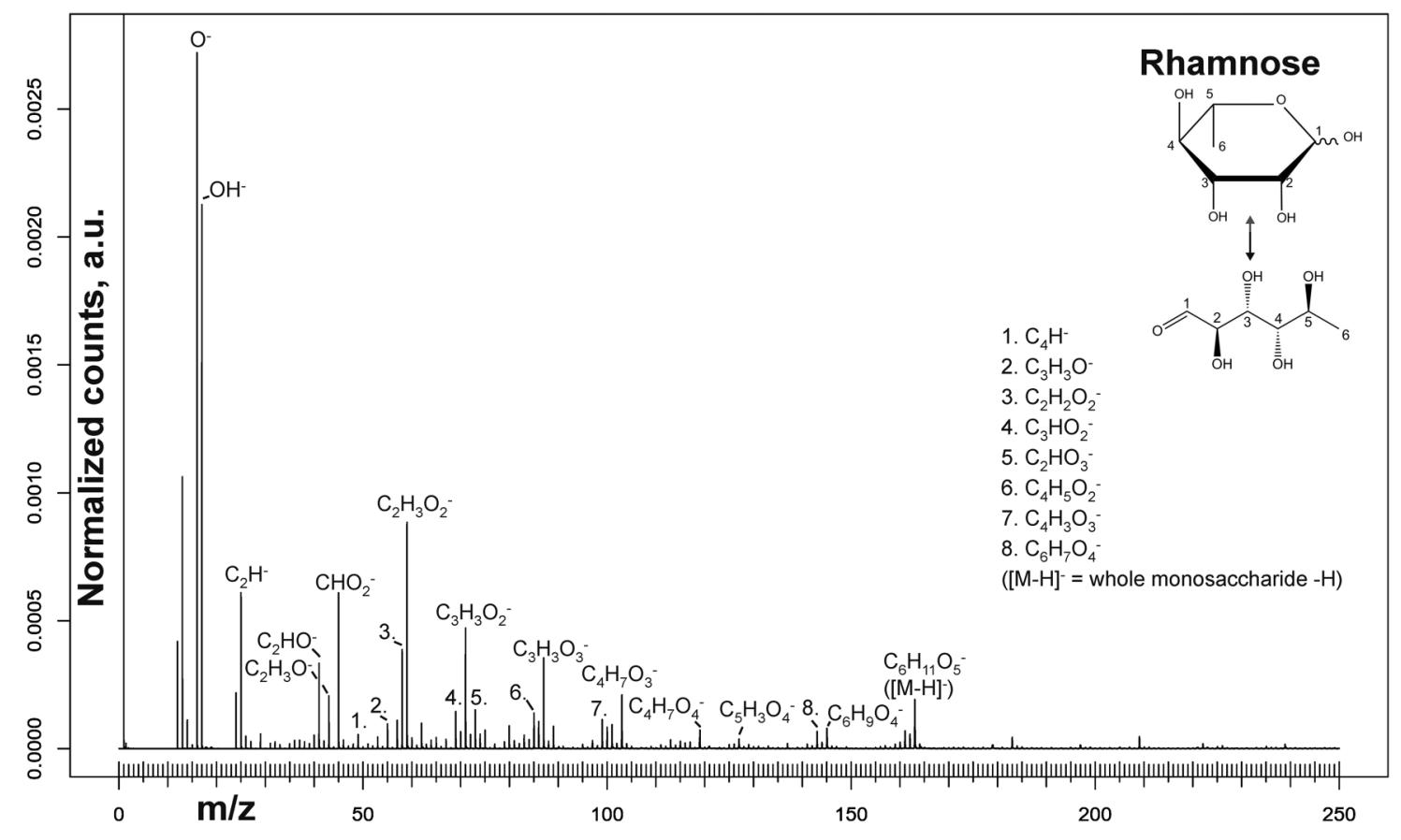

\begin{tabular}{|c|c|}
\hline Accession \# & 01584-01 \\
\hline Host Material: & Silicon wafer \\
\hline घechnique: & SIMS \\
\hline Secondary Source Polarity: & Negative \\
\hline Mass Range: & $0-250 \mathrm{Da}$ \\
\hline $\begin{array}{r}\text { Species Used for Mass } \\
\text { Calibration: }\end{array}$ & $\mathrm{C}^{-}, \mathrm{CH}^{-}, \mathrm{CH}_{2}^{-}, \mathrm{CH}_{3} \mathrm{O}^{-}, \mathrm{C}_{4} \mathrm{H}_{3}^{-}, \mathrm{C}_{3} \mathrm{HO}^{-}, \mathrm{C}_{2} \mathrm{HO}_{2}^{-}$ \\
\hline Primary Ion Dose: & $9.71 \times 10^{11} \mathrm{~cm}^{-2}$ \\
\hline Primary Ion Pulse Width: & $17.2 \mathrm{~ns}$ \\
\hline Pulsed Beam Current: & $0.00076 \mathrm{nA}$ \\
\hline Biological significance: & $\begin{array}{l}\text { L-Rhamnose is a component of the rhamnogalacturonan pectins as } \\
\text { well as of certain bacterial outer cell membranes }\end{array}$ \\
\hline
\end{tabular}




\begin{tabular}{lccc}
\hline & & D-RIBOSE & \\
\hline Spectrum ID \# & Mass (Da) & Species & Peak Assignment \\
\hline 01585 & 71.014 & $\mathrm{C}_{3} \mathrm{H}_{3} \mathrm{O}_{2}^{-}$ & $\mathrm{C} 1$ to $\mathrm{C} 3$ fragment of $\mathrm{M}-\mathrm{H}_{2} \mathrm{O}$ \\
$\ldots$ & 87.009 & $\mathrm{C}_{3} \mathrm{H}_{3} \mathrm{O}_{3}^{-}$ & $\mathrm{C} 1$ to $\mathrm{C}$ fragment of $\mathrm{M}-2 \mathrm{H}$ \\
$\ldots$ & 89.024 & $\mathrm{C}_{3} \mathrm{H}_{5} \mathrm{O}_{3}^{-}$ & $\mathrm{C} 1$ to $\mathrm{C} 3$ fragment of $\mathrm{M}$ \\
$\ldots$ & 99.009 & $\mathrm{C}_{4} \mathrm{H}_{3} \mathrm{O}_{3}^{-}$ & $\mathrm{C} 1$ to $\mathrm{C} 4$ fragment of $\mathrm{M}-\mathrm{H}_{2} \mathrm{O}-2 \mathrm{H}$ \\
$\ldots$ & 129.019 & $\mathrm{C}_{5} \mathrm{H}_{5} \mathrm{O}_{4}^{-}$ & $\mathrm{M}-\left(\mathrm{H}_{2} \mathrm{O}\right)-3 \mathrm{H}$ \\
\hline
\end{tabular}

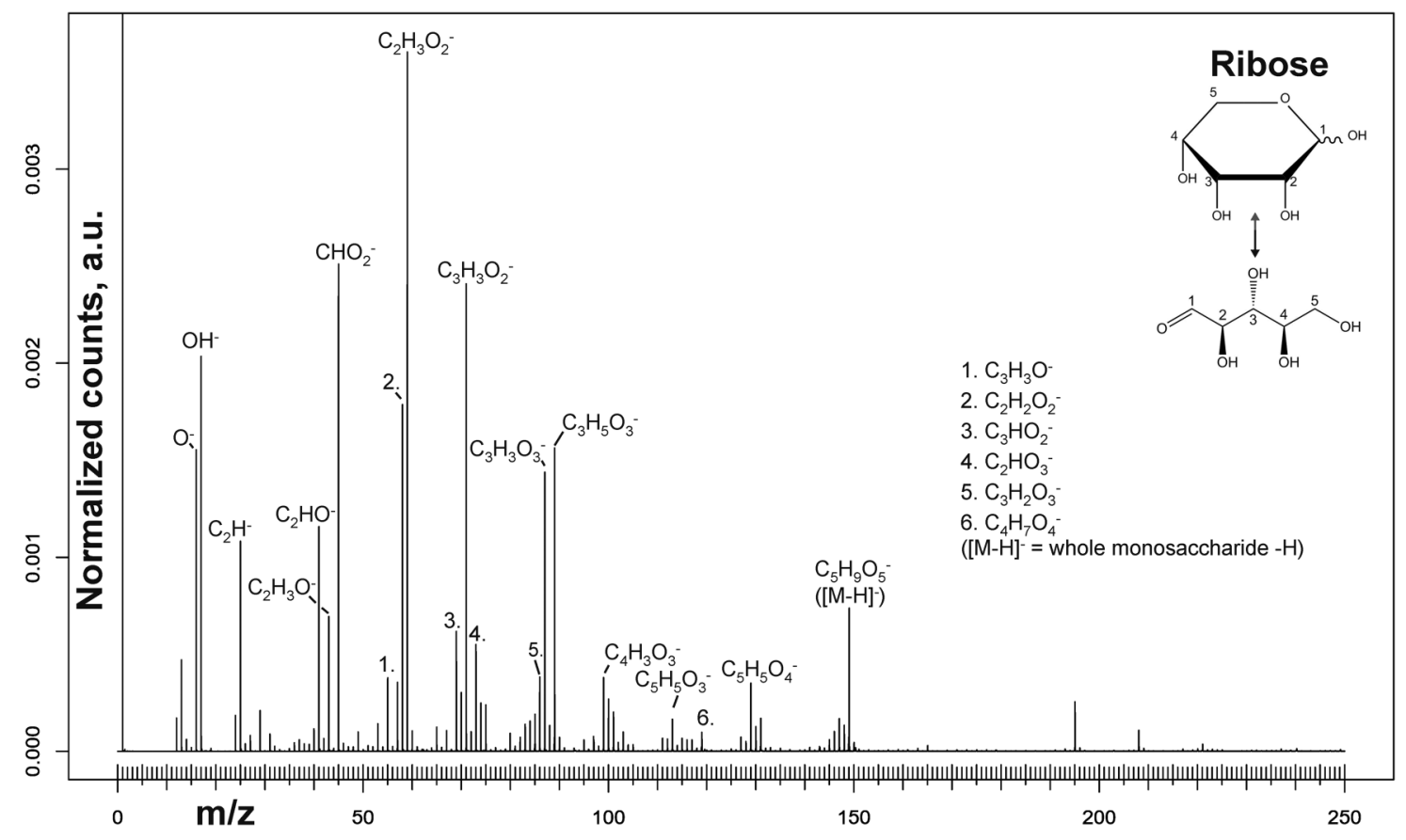

\begin{tabular}{|c|c|}
\hline Accession \# & $01585-01$ \\
\hline Host Material: & Silicon wafer \\
\hline 口 Technique: & SIMS \\
\hline Secondary Source Polarity: & Negative \\
\hline Mass Range: & $0-250 \mathrm{Da}$ \\
\hline $\begin{array}{r}\text { Species Used for Mass } \\
\text { Calibration: }\end{array}$ & $\mathrm{C}^{-}, \mathrm{CH}^{-}, \mathrm{CH}_{2}^{-}, \mathrm{CH}_{3} \mathrm{O}^{-}, \mathrm{C}_{4} \mathrm{H}_{3}^{-}, \mathrm{C}_{3} \mathrm{HO}^{-}, \mathrm{C}_{2} \mathrm{HO}_{2}^{-}$ \\
\hline Primary Ion Dose: & $9.71 \times 10^{11} \mathrm{~cm}^{-2}$ \\
\hline Primary Ion Pulse Width: & $17.2 \mathrm{~ns}$ \\
\hline Pulsed Beam Current: & $0.00076 \mathrm{nA}$ \\
\hline Biological significance: & $\begin{array}{l}\text { D-Ribose (as } \alpha \text {-D-ribofuranose) forms the backbone of ribonucleic } \\
\text { acid and is, among others, part of the molecules adenosine } \\
\text { triphosphate, adenosine diphosphate, and nicotinamide adenine } \\
\text { dinucleotide essential for the metabolism of all living cells }\end{array}$ \\
\hline
\end{tabular}




\begin{tabular}{|c|c|c|c|}
\hline \multicolumn{4}{|c|}{ 2-DEOXY-D-RIBOSE } \\
\hline Spectrum ID \# & Mass (Da) & Species & Peak Assignment \\
\hline 01586 & 71.014 & $\mathrm{C}_{3} \mathrm{H}_{3} \mathrm{O}_{2}^{-}$ & $\mathrm{C} 1$ to $\mathrm{C} 3$ fragment of $\mathrm{M}-\mathrm{H}_{2} \mathrm{O}$ \\
\hline$\ldots$ & 87.009 & $\mathrm{C}_{4} \mathrm{H}_{5} \mathrm{O}_{2}^{-}$ & $\mathrm{C} 1$ to $\mathrm{C} 4$ fragment of $\mathrm{M}-\mathrm{H}_{2} \mathrm{O}$ \\
\hline$\ldots$ & 89.024 & $\mathrm{C}_{3} \mathrm{H}_{5} \mathrm{O}_{3}^{-}$ & $\mathrm{C} 1$ to $\mathrm{C} 3$ fragment of $\mathrm{M}$ \\
\hline$\ldots$ & 97.030 & $\mathrm{C}_{5} \mathrm{H}_{5} \mathrm{O}_{2}^{-}$ & $\mathrm{M}-2\left(\mathrm{H}_{2} \mathrm{O}\right)-\mathrm{H}$ \\
\hline$\ldots$ & 99.009 & $\mathrm{C}_{4} \mathrm{H}_{3} \mathrm{O}_{3}^{-}$ & $\mathrm{C} 1$ to $\mathrm{C} 4$ fragment of $\mathrm{M}-4 \mathrm{H}$ \\
\hline$\ldots$ & 113.024 & $\mathrm{C}_{5} \mathrm{H}_{5} \mathrm{O}_{3}^{-}$ & $\mathrm{M}-\mathrm{H}_{2} \mathrm{O}-3 \mathrm{H}$ \\
\hline$\ldots$ & 115.040 & $\mathrm{C}_{5} \mathrm{H}_{7} \mathrm{O}_{3}{ }^{-}$ & $\mathrm{M}-\mathrm{H}_{2} \mathrm{O}-\mathrm{H}$ \\
\hline$\ldots$ & 119.035 & $\mathrm{C}_{4} \mathrm{H}_{7} \mathrm{O}_{4}^{-}$ & $\ldots$ \\
\hline$\ldots$ & 133.051 & $\mathrm{C}_{5} \mathrm{H}_{9} \mathrm{O}_{4}^{-}$ & $\mathrm{M}-\mathrm{H}$ \\
\hline
\end{tabular}

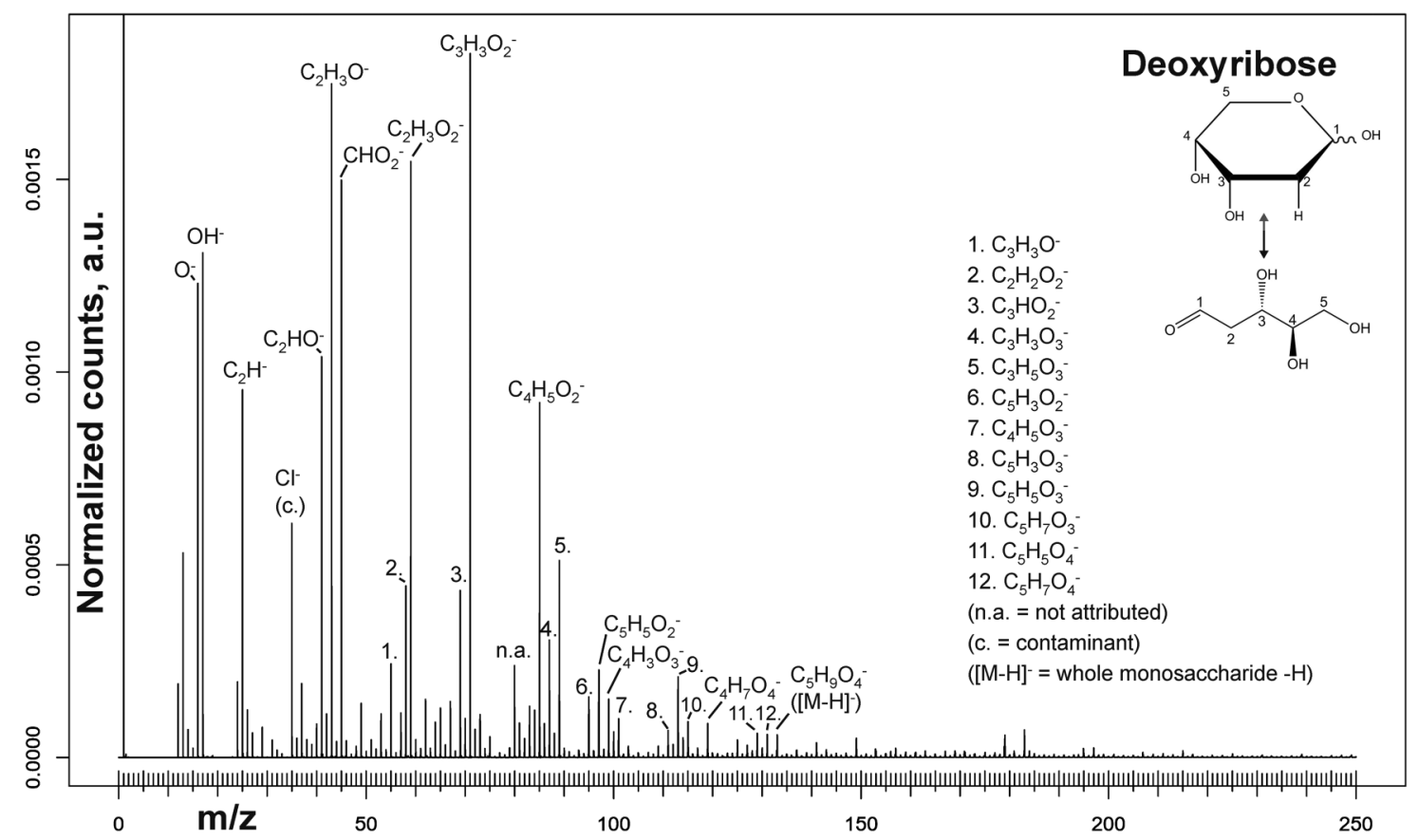

\begin{tabular}{rc}
\hline Accession \# & $01586-01$ \\
\hline Host Material: & Silicon wafer \\
Technique: & SIMS \\
Mass Range: & Negative \\
Secondary Source Polarity: & $0-250 \mathrm{Da}$ \\
Species Used for Mass & $\mathrm{C}^{-}, \mathrm{CH}^{-}, \mathrm{CH}_{2}^{-}, \mathrm{CH}_{3} \mathrm{O}^{-}, \mathrm{C}_{4} \mathrm{H}_{3}^{-}, \mathrm{C}_{3} \mathrm{HO}^{-}, \mathrm{C}_{2} \mathrm{HO}_{2}^{-}$ \\
Calibration: & $9.71 \times 10^{-11} \mathrm{~cm}^{-2}$ \\
Primary lon Dose: & $17.2 \mathrm{~ns}$ \\
Primary lon Pulse Width: & $0.00076 \mathrm{nA}$ \\
Pulsed Beam Current: & 2-Deoxy-D-ribose forms the backbone of deoxyribose nucleic acid \\
Biological significance: &
\end{tabular}




\begin{tabular}{lccc}
\hline & & D-XYLOSE & \\
\hline Spectrum ID \# & Mass (Da) & Species & Peak Assignment \\
\hline 01587 & 71.014 & $\mathrm{C}_{3} \mathrm{H}_{3} \mathrm{O}_{2}^{-}$ & $\mathrm{C} 1$ to $\mathrm{C} 3$ fragment of $\mathrm{M}-\mathrm{H}_{2} \mathrm{O}$ \\
$\ldots$ & 87.009 & $\mathrm{C}_{3} \mathrm{H}_{3} \mathrm{O}_{3}^{-}$ & $\mathrm{C} 1$ to $\mathrm{C}$ fragment of $\mathrm{M}-2 \mathrm{H}$ \\
$\ldots$ & 89.024 & $\mathrm{C}_{3} \mathrm{H}_{5} \mathrm{O}_{3}^{-}$ & $\mathrm{C} 1$ to $\mathrm{C}$ fragment of $\mathrm{M}$ \\
$\ldots$ & 99.009 & $\mathrm{C}_{4} \mathrm{H}_{3} \mathrm{O}_{3}^{-}$ & $\mathrm{C} 1$ to $\mathrm{C} 4$ fragment of $\mathrm{M}-\mathrm{H}_{2} \mathrm{O}-2 \mathrm{H}$ \\
$\ldots$ & 129.019 & $\mathrm{C}_{5} \mathrm{H}_{5} \mathrm{O}_{4}^{-}$ & $\mathrm{M}-\left(\mathrm{H}_{2} \mathrm{O}\right)-3 \mathrm{H}$ \\
$\ldots$ & 149.046 & $\mathrm{C}_{5} \mathrm{H}_{9} \mathrm{O}_{5}^{-}$ & $\mathrm{M}-\mathrm{H}$ \\
$\ldots$ & 299.098 & $\mathrm{C}_{10} \mathrm{H}_{19} \mathrm{O}_{10}^{-}$ & $2 \mathrm{M}-\mathrm{H}$ \\
\hline
\end{tabular}

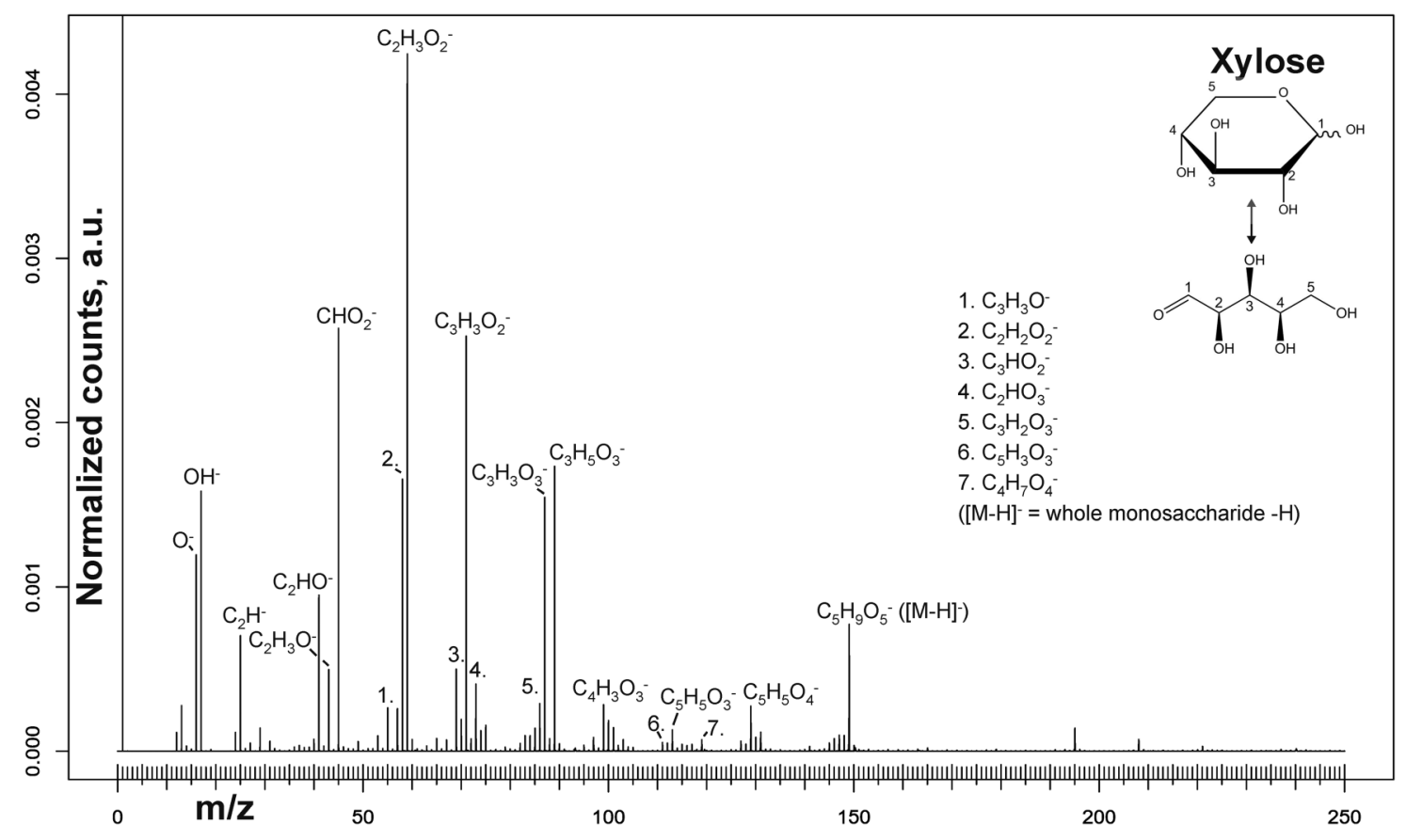

\begin{tabular}{rc}
\hline Accession \# & $01587-01$ \\
\hline Host Material: \\
Technique: & Silicon wafer \\
Mass Range: & SIMS \\
Secondary Source Polarity: & Negative \\
Species Used for Mass & $0-250 \mathrm{Da}$ \\
Calibration: & $\mathrm{C}^{-}, \mathrm{CH}^{-}, \mathrm{CH}_{2}^{-}, \mathrm{CH}_{3} \mathrm{O}^{-}, \mathrm{C}_{4} \mathrm{H}_{3}^{-}, \mathrm{C}_{3} \mathrm{HO}^{-}, \mathrm{C}_{2} \mathrm{HO}_{2}{ }^{-}$ \\
Primary lon Dose: & $9.46 \times 10^{11} \mathrm{~cm}^{-2}$ \\
Primary lon Pulse Width: & $17.2 \mathrm{~ns}$ \\
Pulsed Beam Current: & $0.00074 \mathrm{nA}$ \\
Biological significance: & D-Xylose is the main component of xylan, a highly abundant \\
& hemicellulose, and is, among others, also found as residue in certain \\
& type of pectin \\
\hline
\end{tabular}




\begin{tabular}{|c|c|c|c|}
\hline \multicolumn{4}{|c|}{ N-ACETYL-D-GALACTOSAMINE } \\
\hline Spectrum ID \# & Mass (Da) & Species & Peak Assignment \\
\hline 01588 & 58.030 & $\mathrm{C}_{2} \mathrm{H}_{4} \mathrm{NO}^{-}$ & Amine group \\
\hline$\ldots$ & 71.014 & $\mathrm{C}_{3} \mathrm{H}_{3} \mathrm{O}_{2}^{-}$ & $\mathrm{C} 1$ to $\mathrm{C} 3$ fragment of $\mathrm{M}-\mathrm{NH}_{2}$ \\
\hline$\ldots$ & 87.009 & $\mathrm{C}_{3} \mathrm{H}_{3} \mathrm{O}_{3}^{-}$ & Pyruvate \\
\hline$\ldots$ & 99.001 & $\mathrm{C}_{4} \mathrm{H}_{3} \mathrm{O}_{3}^{-}$ & $\ldots$ \\
\hline$\ldots$ & 100.040 & $\mathrm{C}_{4} \mathrm{H}_{6} \mathrm{NO}_{2}^{-}$ & $\mathrm{C} 1$ to $\mathrm{C} 2$ fragment of $\mathrm{M}$ \\
\hline$\ldots$ & 112.040 & $\mathrm{C}_{5} \mathrm{H}_{6} \mathrm{NO}_{2}^{-}$ & $\mathrm{C} 1$ to $\mathrm{C} 3$ fragment of $\mathrm{M}-\left(\mathrm{H}_{2} \mathrm{O}\right)$ \\
\hline$\cdots$ & 119.035 & $\mathrm{C}_{4} \mathrm{H}_{7} \mathrm{O}_{4}^{-}$ & $\mathrm{C} 3$ to $\mathrm{C} 6$ fragment of $\mathrm{M}-2 \mathrm{H}$ \\
\hline$\ldots$ & 128.035 & $\mathrm{C}_{5} \mathrm{H}_{6} \mathrm{NO}_{3}^{-}$ & $\mathrm{C} 1$ to $\mathrm{C} 3$ fragment of $\mathrm{M}-2 \mathrm{H}$ \\
\hline$\ldots$ & 142.051 & $\mathrm{C}_{6} \mathrm{H}_{8} \mathrm{NO}_{3}^{-}$ & $\mathrm{C} 1$ to $\mathrm{C} 4$ fragment of $\mathrm{M}-\left(\mathrm{H}_{2} \mathrm{O}\right)$ \\
\hline$\ldots$ & 220.083 & $\mathrm{C}_{8} \mathrm{H}_{14} \mathrm{NO}_{6}^{-}$ & $\mathrm{M}-\mathrm{H}$ \\
\hline$\ldots$ & 441.173 & $\mathrm{C}_{16} \mathrm{H}_{29} \mathrm{~N}_{2} \mathrm{O}_{12}^{-}$ & $2 \mathrm{M}-\mathrm{H}$ \\
\hline
\end{tabular}

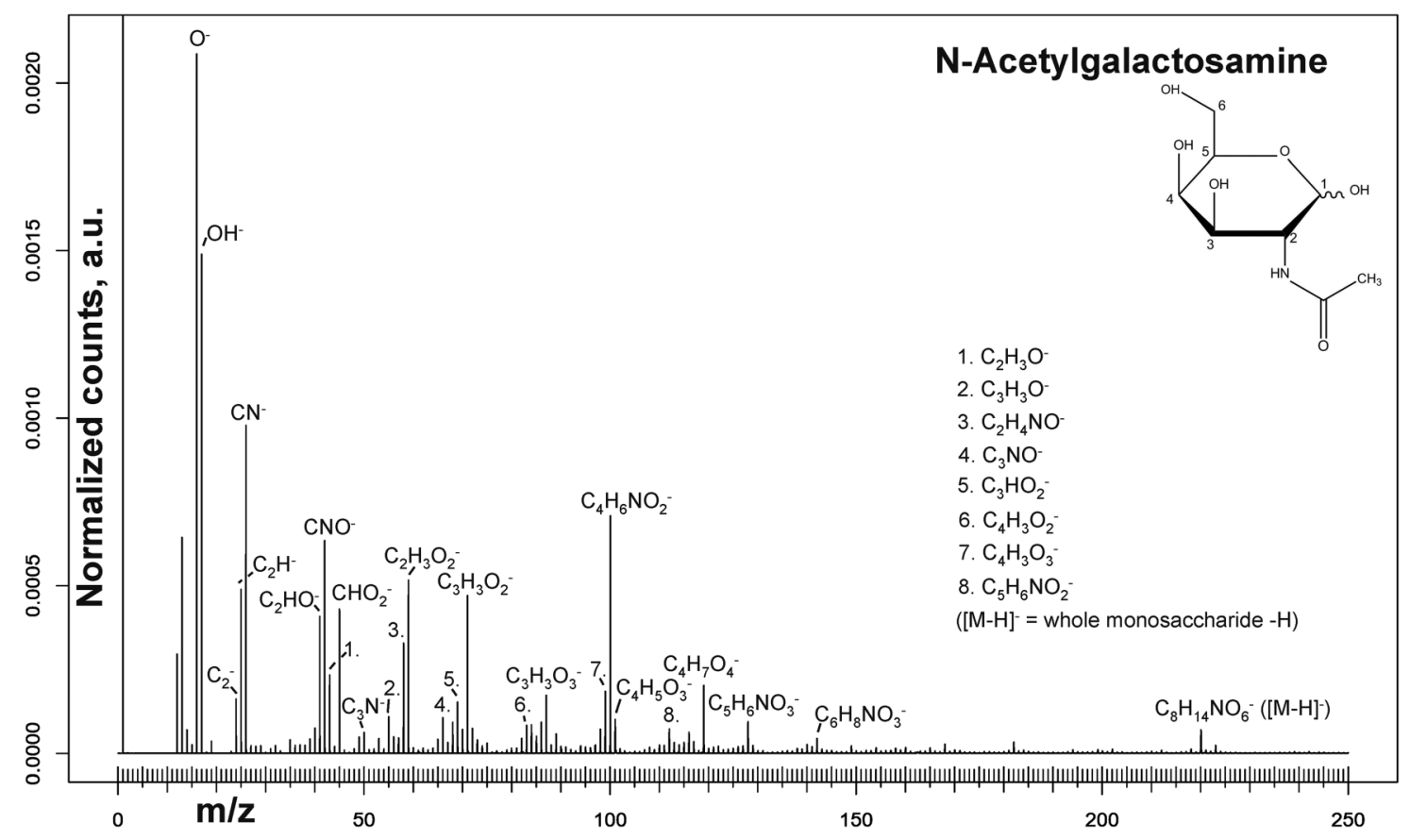

\begin{tabular}{rc}
\hline Accession \# & $\mathbf{0 1 5 8 8 - 0 1}$ \\
\hline Host Material: & Silicon wafer \\
Technique: & SIMS \\
Mass Range: & Negative \\
Secondary Source Polarity: & $0-250 \mathrm{Da}$ \\
Species Used for Mass Calibration: & $\mathrm{C}^{-}, \mathrm{CH}^{-}, \mathrm{CH}_{2}^{-}, \mathrm{CH}_{3} \mathrm{O}^{-}, \mathrm{C}_{4} \mathrm{H}_{3}^{-}, \mathrm{C}_{3} \mathrm{HO}^{-}, \mathrm{C}_{2} \mathrm{HO}_{2}{ }^{-}$ \\
Primary lon Dose: & $9.97 \times 10^{11} \mathrm{~cm}^{-2}$ \\
Primary lon Pulse Width: & $17.2 \mathrm{~ns}$ \\
Pulsed Beam Current: & $0.00078 \mathrm{nA}$ \\
Biological significance: & $N$-acetylgalactosamine is found in a wide range of mucoproteins, \\
& where it often serves as a linking point between the protein \\
& backbone and its covalently attached polysaccharide chains \\
\hline
\end{tabular}




\begin{tabular}{|c|c|c|c|}
\hline \multicolumn{4}{|c|}{ N-ACETYL-D-GLUCOSAMINE } \\
\hline Spectrum ID \# & Mass (Da) & Species & Peak Assignment \\
\hline 01589 & 58.030 & $\mathrm{C}_{2} \mathrm{H}_{4} \mathrm{NO}^{-}$ & Amide \\
\hline$\ldots$ & 71.014 & $\mathrm{C}_{3} \mathrm{H}_{3} \mathrm{O}_{2}^{-}$ & C1 to $\mathrm{C} 3$ fragment of $\mathrm{M}-\mathrm{NH}_{2}$ \\
\hline$\ldots$ & 87.009 & $\mathrm{C}_{3} \mathrm{H}_{3} \mathrm{O}_{3}^{-}$ & Pyruvate \\
\hline$\ldots$ & 99.001 & $\mathrm{C}_{4} \mathrm{H}_{3} \mathrm{O}_{3}^{-}$ & $\ldots$ \\
\hline$\ldots$ & 100.040 & $\mathrm{C}_{4} \mathrm{H}_{6} \mathrm{NO}_{2}^{-}$ & C1 to $\mathrm{C} 2$ fragment of $\mathrm{M}$ \\
\hline$\cdots$ & 112.040 & $\mathrm{C}_{5} \mathrm{H}_{6} \mathrm{NO}_{2}^{-}$ & C1 to $\mathrm{C} 3$ fragment of $\mathrm{M}-\left(\mathrm{H}_{2} \mathrm{O}\right)$ \\
\hline$\cdots$ & 119.035 & $\mathrm{C}_{4} \mathrm{H}_{7} \mathrm{O}_{4}^{-}$ & $\mathrm{C} 3$ to $\mathrm{C} 6$ fragment of $\mathrm{M}-2 \mathrm{H}$ \\
\hline$\ldots$ & 128.035 & $\mathrm{C}_{5} \mathrm{H}_{6} \mathrm{NO}_{3}^{-}$ & $\mathrm{C} 1$ to $\mathrm{C} 3$ fragment of $\mathrm{M}-2 \mathrm{H}$ \\
\hline$\ldots$ & 142.051 & $\mathrm{C}_{6} \mathrm{H}_{8} \mathrm{NO}_{3}^{-}$ & $\mathrm{C} 1$ to $\mathrm{C} 4$ fragment of $\mathrm{M}-\left(\mathrm{H}_{2} \mathrm{O}\right)$ \\
\hline$\ldots$ & 160.062 & $\mathrm{C}_{6} \mathrm{H}_{10} \mathrm{NO}_{4}^{-}$ & $\mathrm{C} 1$ to $\mathrm{C} 4$ fragment of $\mathrm{M}$ \\
\hline$\ldots$ & 220.083 & $\mathrm{C}_{8} \mathrm{H}_{14} \mathrm{NO}_{6}^{-}$ & $\mathrm{M}-\mathrm{H}$ \\
\hline$\cdots$ & 441.173 & $\mathrm{C}_{16} \mathrm{H}_{29} \mathrm{~N}_{2} \mathrm{O}_{12}^{-}$ & $2 \mathrm{M}-\mathrm{H}$ \\
\hline
\end{tabular}

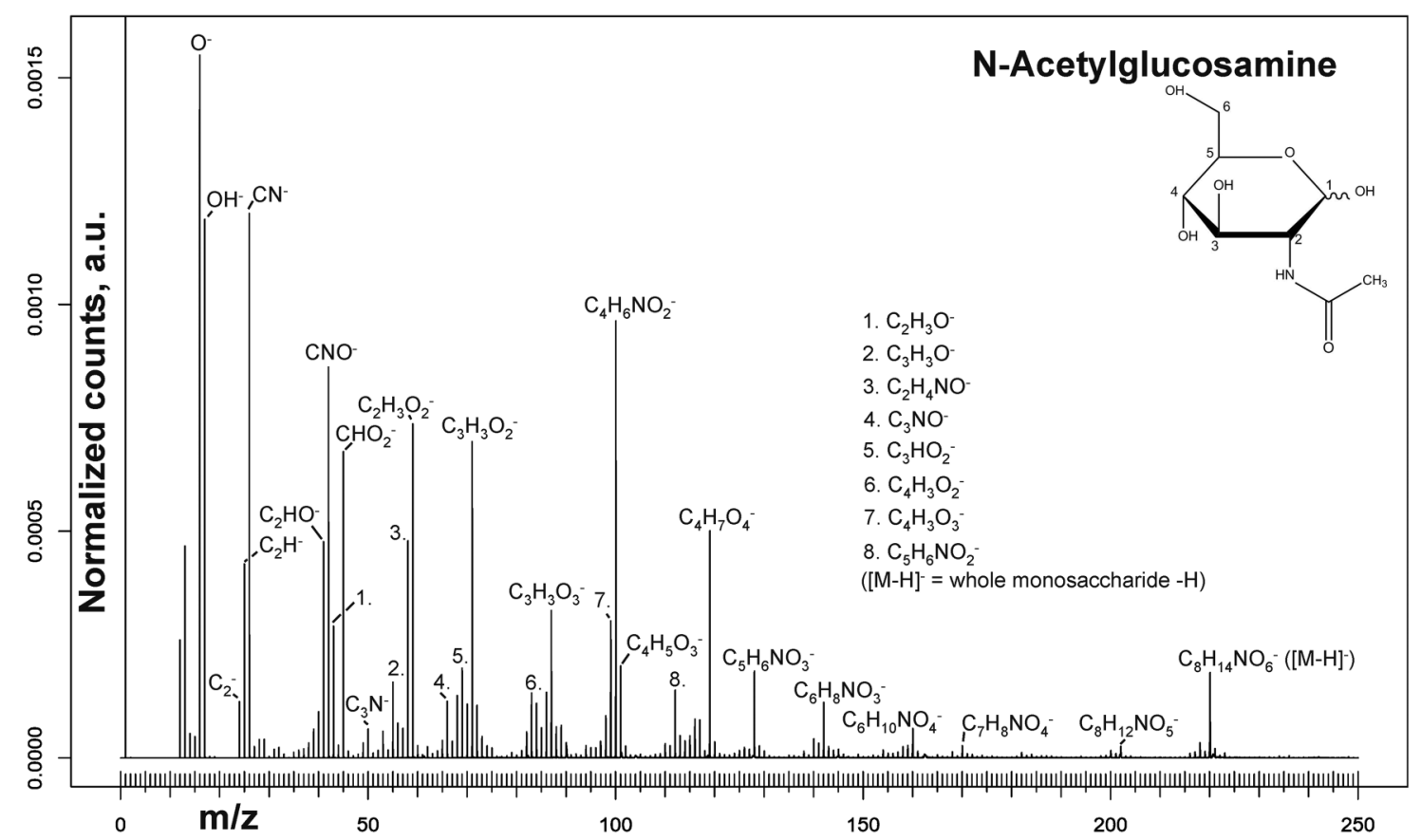

\begin{tabular}{|c|c|}
\hline Accession \# & 01589-01 \\
\hline - Host Material: & Silicon wafer \\
\hline Technique: & SIMS \\
\hline Secondary Source Polarity: & Negative \\
\hline Mass Range: & $0-250 \mathrm{Da}$ \\
\hline $\begin{array}{r}\text { Species Used for Mass } \\
\text { Calibration: }\end{array}$ & $\mathrm{C}^{-}, \mathrm{CH}^{-}, \mathrm{CH}_{2}^{-}, \mathrm{CH}_{3} \mathrm{O}^{-}, \mathrm{C}_{4} \mathrm{H}_{3}^{-}, \mathrm{C}_{3} \mathrm{HO}^{-}, \mathrm{C}_{2} \mathrm{HO}_{2}^{-}$ \\
\hline Primary lon Dose: & $9.46 \times 10^{11} \mathrm{~cm}^{-2}$ \\
\hline Primary lon Pulse Width: & $17.2 \mathrm{~ns}$ \\
\hline Pulsed Beam Current: & $0.00074 \mathrm{nA}$ \\
\hline Biological significance: & $\begin{array}{l}N \text {-acetyl-D-glucosamine is the monomer unit of chitin, a polysaccharide } \\
\text { that is, among others, the primary components of arthropodal } \\
\text { exoskeletons and fungal cell walls. Independently, alternating covalently } \\
\text { bound } N \text {-acetyl-D-glucosamine and } N \text {-acetylmuramic acid units form the } \\
\text { amino sugar chains in the peptidoglycan, the bacterial cell wall. } \\
N \text {-acetyl-D-glucosamine is also found, among others, in mucoproteins }\end{array}$ \\
\hline
\end{tabular}




\section{N-ACETYLMURAMIC ACID}

\begin{tabular}{lccc}
\hline Spectrum ID \# & Mass (Da) & Species & Peak Assignment \\
\hline 01590 & 58.030 & $\mathrm{C}_{2} \mathrm{H}_{4} \mathrm{NO}^{-}$ & Amide group \\
$\ldots$ & 71.014 & $\mathrm{C}_{3} \mathrm{H}_{3} \mathrm{O}_{2}^{-}$ & $\ldots$ \\
$\ldots$ & 87.009 & $\mathrm{C}_{3} \mathrm{H}_{3} \mathrm{O}_{3}{ }^{-}$ & Pyruvate \\
$\ldots$ & 89.024 & $\mathrm{C}_{3} \mathrm{H}_{5} \mathrm{O}_{3}^{-}$ & Ethylcarboxylate \\
$\ldots$ & 100.040 & $\mathrm{C}_{4} \mathrm{H}_{6} \mathrm{NO}_{2}^{-}$ & $\mathrm{C} 1$ to C2 + amide group fragment of $\mathrm{M}$ \\
$\ldots$ & 112.040 & $\mathrm{C}_{5} \mathrm{H}_{6} \mathrm{NO}_{2}^{-}$ & $\mathrm{C} 1$ to C3 fragment of $\mathrm{M}-$ ethylcarboxylate $-\mathrm{H}$ \\
$\ldots$ & 142.051 & $\mathrm{C}_{6} \mathrm{H}_{8} \mathrm{NO}_{3}^{-}$ & C1 to C4 fragment of $\mathrm{M}-$ ethylcarboxylate $-\mathrm{H}$ \\
$\ldots$ & 292.104 & $\mathrm{C}_{11} \mathrm{H}_{18} \mathrm{NO}_{8}^{-}$ & $\mathrm{M}-\mathrm{H}$ \\
$\ldots$ & 585.215 & $\mathrm{C}_{22} \mathrm{H}_{37} \mathrm{~N}_{2} \mathrm{O}_{16}^{-}$ & $2 \mathrm{M}-\mathrm{H}$ \\
\hline
\end{tabular}

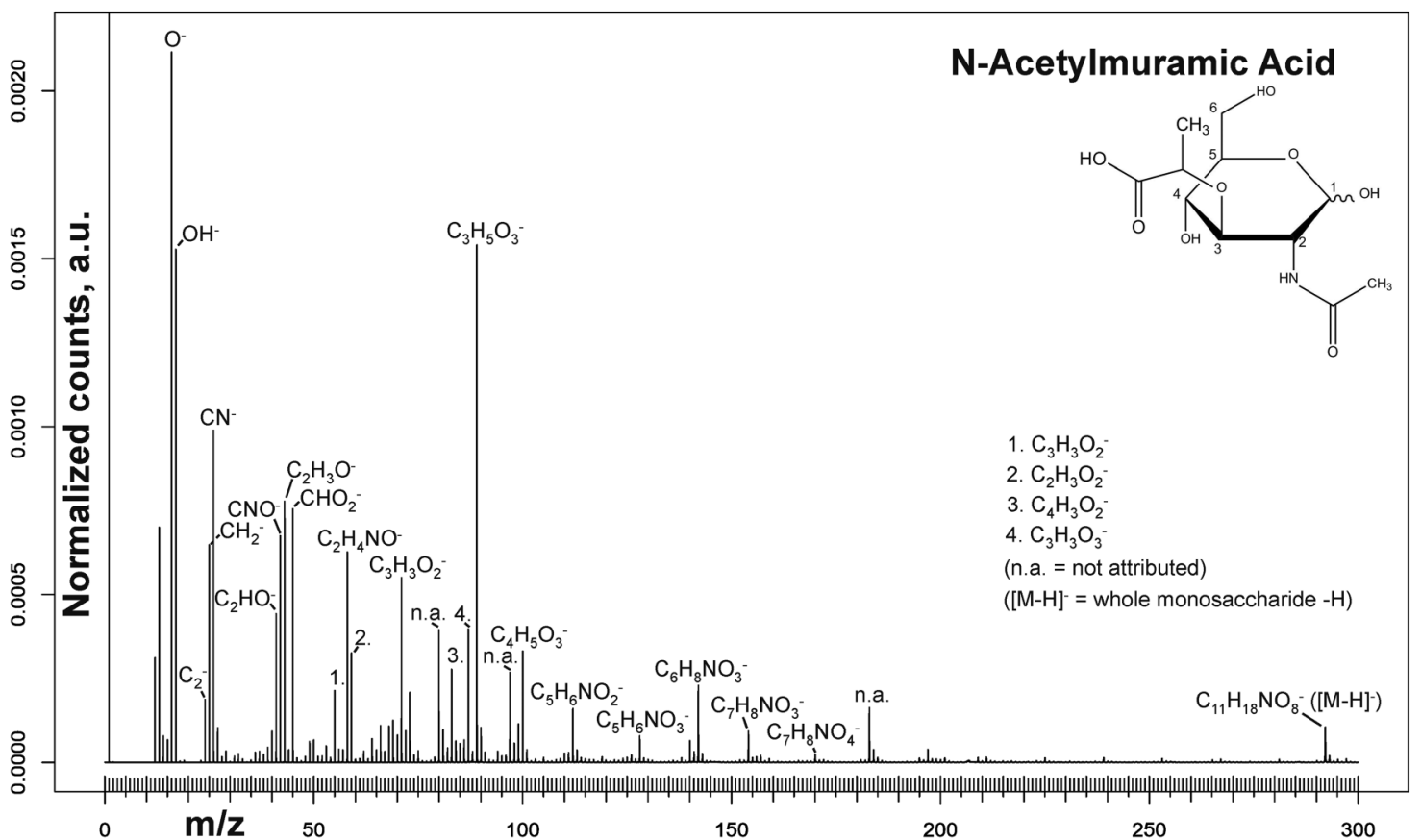

\begin{tabular}{|c|c|}
\hline Accession \# & $01590-01$ \\
\hline Host Material: & Silicon wafer \\
\hline 口 Technique: & SIMS \\
\hline Secondary Source Polarity: & Negative \\
\hline Mass Range: & 0-300 Da \\
\hline $\begin{array}{r}\text { Species Used for Mass } \\
\text { Calibration: }\end{array}$ & $\mathrm{C}^{-}, \mathrm{CH}^{-}, \mathrm{CH}_{2}^{-}, \mathrm{CH}_{3} \mathrm{O}^{-}, \mathrm{C}_{4} \mathrm{H}_{3}^{-}, \mathrm{C}_{3} \mathrm{HO}^{-}, \mathrm{C}_{2} \mathrm{HO}_{2}^{-}$ \\
\hline Primary lon Dose: & $9.46 \times 10^{11} \mathrm{~cm}^{-2}$ \\
\hline Primary lon Pulse Width: & $17.2 \mathrm{~ns}$ \\
\hline Pulsed Beam Current: & $0.00074 \mathrm{nA}$ \\
\hline Biological significance: & $\begin{array}{l}\text { Alternating covalently bound } N \text {-acetylmuramic acid and } \\
N \text {-acetyl-D-glucosamine units form the amino sugar chains of the } \\
\text { peptidoglycan, the bacterial cell wall }\end{array}$ \\
\hline
\end{tabular}




\begin{tabular}{lrcc}
\hline \multicolumn{3}{c}{ N-ACETYLNEURAMINIC ACID } \\
\hline Spectrum ID \# & Mass (Da) & Species & Peak Assignment \\
\hline 01591 & 58.030 & $\mathrm{C}_{2} \mathrm{H}_{4} \mathrm{NO}^{-}$ & Amide group \\
$\ldots$ & 71.014 & $\mathrm{C}_{3} \mathrm{H}_{3} \mathrm{O}_{2}^{-}$ & $\mathrm{C} 1$ to $\mathrm{C}$ fragment of $\mathrm{M}-$ methyl carboxylate $-\mathrm{H}$ \\
$\ldots$ & 72.993 & $\mathrm{C}_{2} \mathrm{HO}_{3}^{-}$ & $\mathrm{C} 1$ with its $(\mathrm{OH})$ and $(\mathrm{COOH})$ groups $-\mathrm{H}$ \\
$\ldots$ & 87.009 & $\mathrm{C}_{3} \mathrm{H}_{3} \mathrm{O}_{3}^{-}$ & Linear triol at $\mathrm{C} 5$ \\
$\ldots$ & 100.040 & $\mathrm{C}_{4} \mathrm{H}_{6} \mathrm{NO}_{2}^{-}$ & $\mathrm{C} 4$ to $\mathrm{C} 5+\mathrm{O}$ fragment of $\mathrm{M}-$ linear triol $-\mathrm{H}$ \\
$\ldots$ & 115.004 & $\mathrm{C}_{4} \mathrm{H}_{3} \mathrm{O}_{4}^{-}$ & $\mathrm{C} 1$ to $\mathrm{C}$ fragment of $\mathrm{M}-2 \mathrm{H}$ \\
$\ldots$ & 126.056 & $\mathrm{C}_{6} \mathrm{H}_{8} \mathrm{NO}_{2}^{-}$ & $\ldots$ \\
$\ldots$ & 170.046 & $\mathrm{C}_{7} \mathrm{H}_{8} \mathrm{NO}_{4}^{-}$ & $\mathrm{C} 4$ to $\mathrm{C} 5+\mathrm{O}$ fragment of M $-\left(\mathrm{H}_{2} \mathrm{O}\right)$ \\
\hline
\end{tabular}

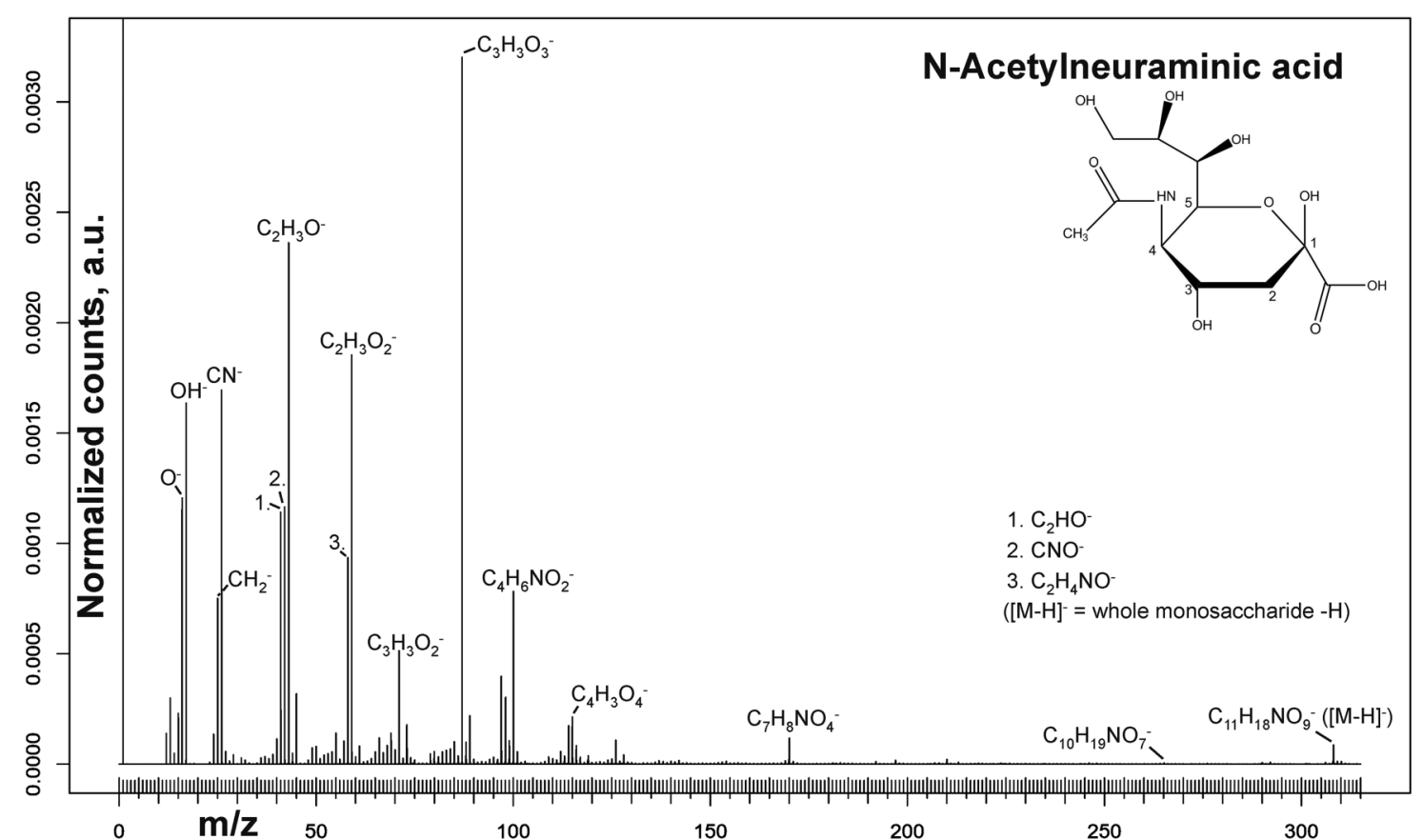

\begin{tabular}{|c|c|}
\hline Accession \# & 01591-01 \\
\hline Host Material: & Silicon wafer \\
\hline Technique: & SIMS \\
\hline Secondary Source Polarity: & Negative \\
\hline Mass Range: & $0-315 \mathrm{Da}$ \\
\hline $\begin{array}{r}\text { Species Used for Mass } \\
\text { Calibration: }\end{array}$ & $\mathrm{C}^{-}, \mathrm{CH}^{-}, \mathrm{CH}_{2}^{-}, \mathrm{CH}_{3} \mathrm{O}^{-}, \mathrm{C}_{4} \mathrm{H}_{3}^{-}, \mathrm{C}_{3} \mathrm{HO}^{-}, \mathrm{C}_{2} \mathrm{HO}_{2}^{-}$ \\
\hline Primary lon Dose: & $9.97 \times 10^{11} \mathrm{~cm}^{-2}$ \\
\hline Primary Ion Pulse Width: & $17.2 \mathrm{~ns}$ \\
\hline Pulsed Beam Current: & $0.00078 \mathrm{nA}$ \\
\hline Biological significance: & $\begin{array}{c}N \text {-acetylneuraminic acid is found in a wide range of glycoproteins } \\
\text { and is crucial in the modulation of neurosynaptic transmission in } \\
\text { humans }\end{array}$ \\
\hline
\end{tabular}



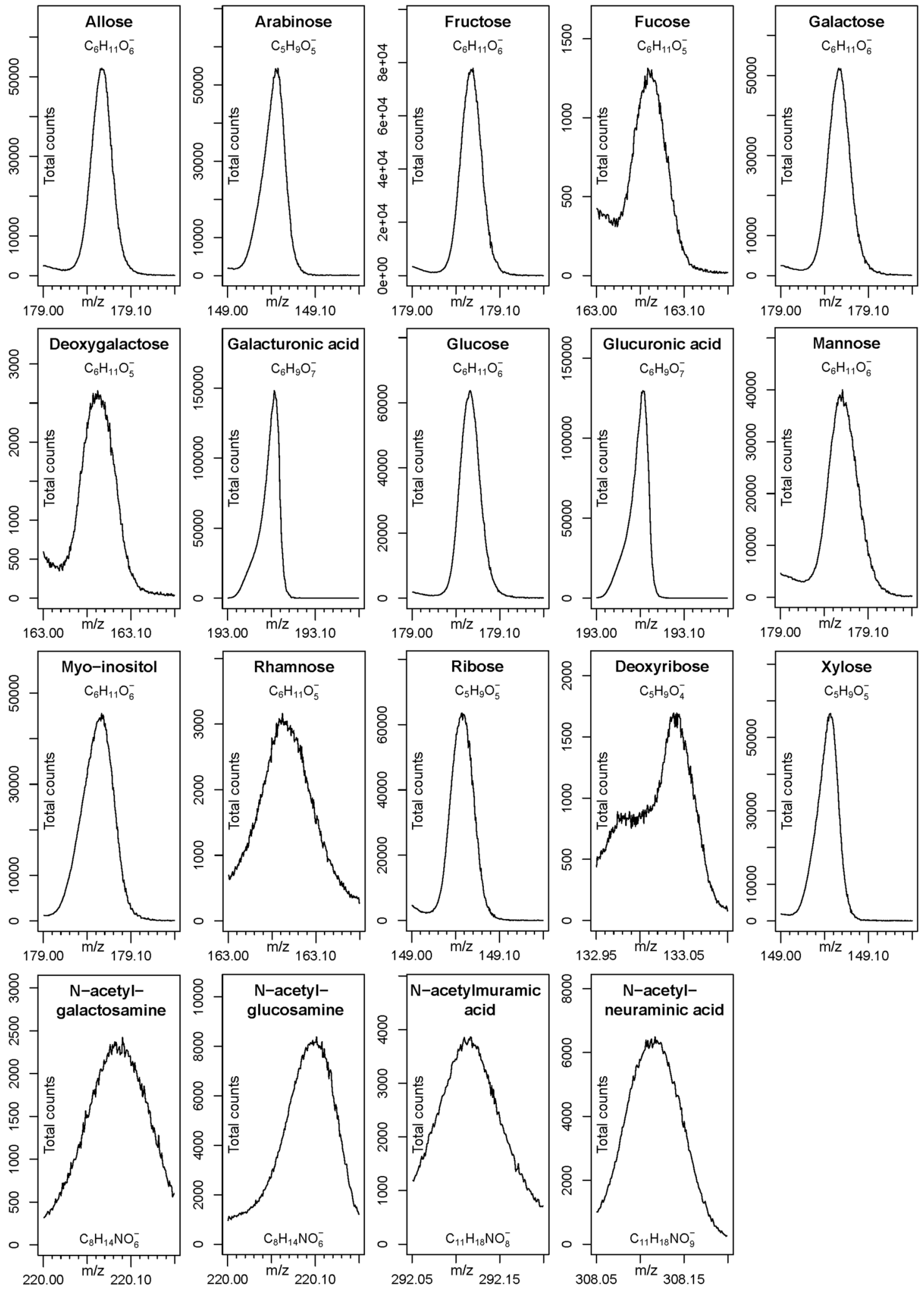

Accession \# 01573-01, 01574-01, 01575-01, 01576-01, 01577-01, 01578-01, 01579-01, 01580-01, 01581-01, 01582-01, 01583-01, 01584-01, 01585-01, 01586-01, 01587-01, 01588-01, 01589-01, 01590-01, 01591-01 